\title{
REAL-TIME HOSTILE ATTRIBUTION MEASUREMENT AND AGGRESSION IN CHILDREN
}

\author{
by \\ ANNA YAROS \\ JOHN E. LOCHMAN, COMMITTEE CHAIR \\ MARK R. KLINGER \\ ROSANNA E. GUADAGNO \\ MELISSA F. JACKSON \\ JOAN M. BARTH
}

\begin{abstract}
A DISSERTATION
Submitted in partial fulfillment of the requirements for the degree of Doctor of Philosophy in the Department of Psychology in the Graduate School of The University of Alabama
\end{abstract}

TUSCALOOSA, ALABAMA 
Copyright Anna Yaros 2012

ALL RIGHTS RESERVED 


\begin{abstract}
Hostile attributions are acknowledged as an important part of maladaptive social information-processing that results in aggression in children. Literature suggests the largest effect size between aggressive behavior and hostile attributions is found when hostile attributions are measured using staged situations rather than commonly used vignette-based measures (Orobio de Castro et al., 2002), but few, if any, studies have investigated hostile attributions as they occur in real-time. The current study uses an interactive, video racing game along with a verbal and nonverbal response procedure to measure hostile attributions while children are playing against a presumed peer. A sample of 75 children between the ages of 10 and 13 played the game while their parents were asked to rate their child's reactive and proactive aggression. It was expected that the new real-time measure of hostile attributions would predict reactive aggression, but not proactive aggression. The predictive ability of the new measure was compared with that of the traditional, vignette-based measure of hostile attributions.

Participants played a computer video game created for this study by racing their car against a presumed peer, though the opponent's car was actually computer-operated and preprogrammed to ambiguously provoke participants by crashing into their car. A visual prompt appeared on screen to elicit real-time attribution responses, either verbally or nonverbally, after each of eight crashes and at eight non-crash moments.
\end{abstract}


Participants' hostile attributions using the nonverbal and verbal response procedures were significantly positively related to parent-rated reactive aggression, when controlling for proactive aggression. Multiple regression indicated that the real-time measure was a significant predictor of reactive aggression, while the vignette-based measure was not. The results of this study illustrate how hostile attributions in real-time occur quickly and simultaneously with social interaction, which differs from the deliberative, controlled appraisal process described in previous literature. These findings can be used to identify specific appraisal deficits in children at risk for aggressive behavior, which could enhance preventive interventions by more precisely targeting these cognitions and more effectively preventing aggression. 


\section{LIST OF ABBREVIATIONS AND SYMBOLS}

$\alpha$

$\beta$

$\Delta$

F

$M$

$n$

$p$

$r$

$\mathrm{R}^{2}$

$S D$

$t$

$<$

$=$

Cronbach's index of internal consistency; probability of a Type I error

Standardized beta coefficient

Change in statistic's value

Fisher's $F$ ratio

Mean

Number of cases in subsample

Probability associated with the occurrence under the null hypothesis of a value as extreme as or more extreme than the observed value

Pearson product-moment correlation

Variability explained by independent variables in a regression model

Standard deviation

Computed value of $t$-test

Less than

Equal to 


\section{ACKNOWLEDGMENTS}

I submit this dissertation with great appreciation to a number of people, without whom this would not be possible. First, I sincerely thank John Lochman, advisor and committee chair, for the hours and effort he has dedicated to this project. In addition, his knowledge and guidance has been invaluable at each step in my graduate school career. I am grateful for the many times he has gone above and beyond that which was necessary to assist me as I navigated my way through my doctoral degree. I would also like to thank my committee members, Mark Klinger, Melissa Jackson, Rosanna Guadagno, and Joan Barth. I am lucky that each member has contributed a unique expertise to this project and I appreciate the suggestions and recommendations that have undoubtedly improved my dissertation. I owe special thanks to Tonio Loewald, who worked with me to understand my vision for the video game in this study and whose skill and generosity allowed it to come to fruition.

A number of other people deserve thanks for helping with bits and pieces of this project along the way, including the entire staff of the UA Center for the Prevention of Youth Behavior Problems (especially Shanta Burrell), Jill Rosenbaum, Alberto Jimenez-Camargo, Rachel Baden, Laura Larsen, Denicia Titchner, Amanda Sockwell, and the UA Department of Psychology staff.

I also want to express my appreciation to all the friends and colleagues who have completed this journey with me, especially my "academic twin”, Mary Wojnaroski. To them, and to my parents and my family, who provided love, kind words, and faith, and who understood when I said "I have to work on my dissertation," I am forever grateful. 


\section{CONTENTS}

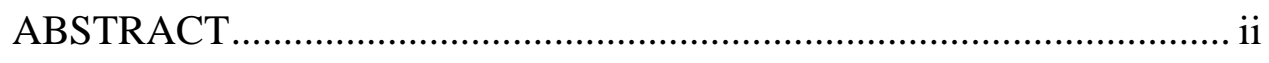

LIST OF ABBREVIATIONS AND SYMBOLS .............................. iv ACKNOWLEDGMENTS ..............................................................

LIST OF TABLES ................................................................ vii

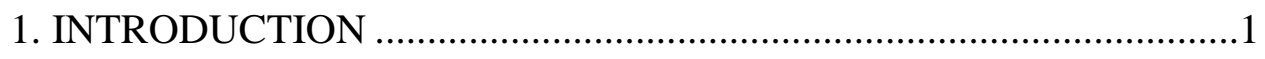

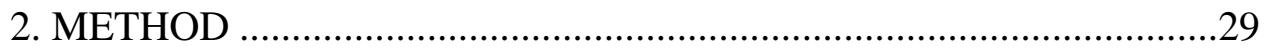

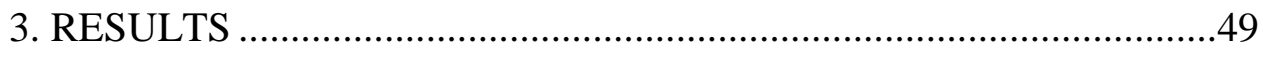

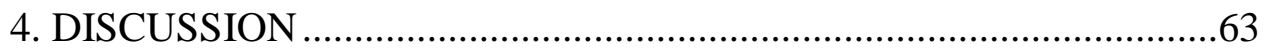

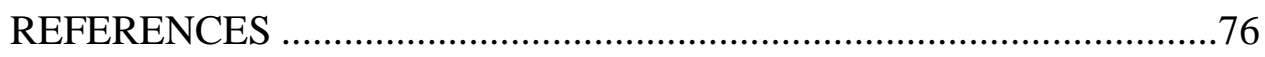

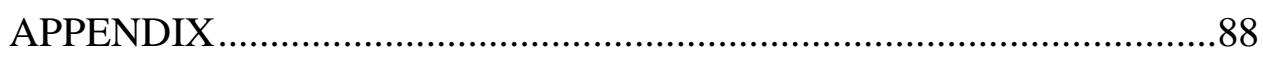




\section{LIST OF TABLES}

1. Real-time Verbal Response Coding Scheme ..................................................41

2. Hostile Attribution Bias (HAB) Vignette Measure Coding Scheme..................45

3. Means and Standard Deviations for Variables ...............................................50

4. Zero-order Correlations between Independent and Dependent Variables........55

5. Hypothesis 3: Sequential Set Regressions..................................57

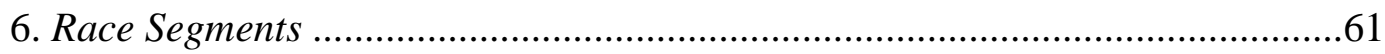




\section{CHAPTER 1}

\section{INTRODUCTION}

Research on aggression has provided evidence that certain cognitive processes, especially those at work during social interactions, reliably predict aggressive behavior. In particular, social cognitive theory has been used as an etiological framework for understanding aggression in children (Crick \& Dodge, 1994, 1996; Dodge \& Coie, 1987; Lochman \& Dodge, 1994; Perry, Perry, \& Rasmussen, 1986). Social cognitions related to aggressive behavior in children include hostile attributions of intent (see Orobio de Castro, Veerman, Koops, Bosch, \& Monshouwer, 2002 for a review), normative beliefs (e.g. Huesmann \& Guerra, 1997), outcome expectations (e.g. Lochman \& Dodge, 1994), self-efficacy (e.g. Erdley \& Asher, 1996), perceived competence (e.g. Lochman \& Dodge, 1994; Kupersmidt \& Patterson, 1991), and response evaluation (e.g. Crick \& Ladd, 1990). These are examples of maladaptive problem-solving and decision-making processes that result in aggressive behavior, many of which have been consolidated into one model of social cognition.

\section{Social Information-Processing Model}

The social cognitive theory that has been most widely used to explain child aggression is Crick \& Dodge's (1994) social information-processing (SIP) model. The social information- 
processing model explains aggressive acts as the outcome of a set of cognitive processes that integrates information from one's surroundings with one's internal cognitive style (Crick \& Dodge, 1994; Dodge, 2006). The six steps of the model begin at (1) encoding and (2) interpreting situational cues, followed by (3) clarifying one's personal goals, and (4) developing, (5) evaluating, and (6) enacting a behavioral response (Crick \& Dodge, 1994). The model has provided structure for investigations of social cognitive styles that relate to aggression in children. Research has examined the six steps of the model on various levels including as one unified model and as steps that encompass other, smaller processes (e.g. Fontaine \& Dodge, 2006).

The model asserts that maladaptive processing at each step could lead to aggressive behavior. This is evidenced in the relation between aggression in children and problems at each SIP step including (1) attending to more aggressive cues (Dodge, Lochman, Harnish, Bates, \& Pettit, 1997), (2) interpreting situational cues as more hostile (Orobio de Castro et al., 2002), (3) setting more aggressive social goals (e.g. Erdley \& Asher, 1996), (4) thinking of fewer prosocial behavioral responses (e.g. Lochman \& Dodge, 1984), and (5) expecting aggressive responses to be more successful (e.g. Crick \& Dodge, 1996).

For the current study, the second step of the model, interpretation of cues, is the focus of the investigation (Crick \& Dodge, 1994). According to the SIP model, interpretation of cues includes attribution formation as well as other interpretative processes such as making evaluative judgments about a social interaction (Crick \& Dodge, 1994). These judgments are thought to be based on a number of factors, especially past experiences. A child's previous social interactions and relationships influence his or her internal emotions and reasoning. Each of these factors also 
shapes the way children process social information, including how they make attributions. This project centers on the study of how attributions are formed and measured, which is a specific component of step 2 of the SIP model. While this portion of step 2 is the central construct of this research, it is viewed in the context of the SIP model as a whole and its effect on aggressive behavior. This study addresses how attributions are formed and measured using both real-time, or immediate, processing and off-line, or delayed, processing.

\section{On-line vs. Off-line Processing of Social Information}

Processing in real time, or on-line processing, undoubtedly plays a significant role in social information-processing, though research has not often focused on this aspect of social cognition (Crick \& Dodge, 1994; Fontaine, 2008; Fontaine \& Dodge, 2006; Milch-Reich, Campbell, Pelham, Connelly, \& Geva, 1999). On-line processing can be defined as real-time, immediate, and "in the moment" mental processing (Fontaine, 2008; Fontaine \& Dodge, 2006; Milch-Reich et al., 1999). A key distinction between on-line and off-line processing is that the former refers to the cognitions during a social interaction or event, while the latter involves thoughts that exist before and after the event. For the purposes of this study, real-time and offline processing denote the point in time that processing occurs in relation to the occurrence of an event.

Within the explanation of the SIP model, Crick and Dodge (1994) outline the need for additional studies of the real-time aspects of social information-processing. They noted that online, or real-time, processing involves the retrieval of information from the long-term memory. They explained that in the moment that this processing occurs, it is unclear to what extent children are aware and deliberative of their thoughts. In this sense, real-time social information- 
processing probably includes both controlled and automatic thoughts (Dodge, 2008), though this is hard to capture. It has been noted that research methods used in studying social informationprocessing may not effectively measure social cognitions due to the automatic nature of some information-processing (Crick \& Dodge, 1994; Fontaine, 2008). More recent research has sought to expand the capabilities of methodology to measure real-time processing.

On-line processing models that include immediate social cognitions have been applied directly to several steps of the SIP model. At the first step of the SIP model, encoding, MilchReich and colleagues (1999) investigated on-line processing using verbal protocols, or verbal descriptions, of pictures to see if children encoded critical cues and integrated different concepts in the pictures. Descriptions of social situations portrayed in pictures were elicited from the children as they saw the pictures for the first time. This allowed researchers to acquire real-time, immediate information about encoding. As part of their responses, some children speculated about inferential or causal links between parts of the picture, which indicated that these children had begun moving on to the second SIP stage, interpretation of cues. In spite of this, few studies have explicitly assessed real-time interpretation of social cues in social situations.

Several recent studies have furthered this investigation of real-time encoding using innovative methods. Young (2010) utilized a reaction time task to measure how children encoded angry faces versus happy faces. This reaction time measure allowed her to examine selective attention bias toward aggressive cues in real-time. Other researchers have recently used eyetracking to determine which cues aggressive children attend to (Horsley, Orobio de Castro, \& Van der Schoot, 2010). As children looked at specific parts of a picture for longer, it was inferred that they were paying more attention to the cues in that part of the picture. While the 
authors (Horsley et al., 2010) suggested that hostile attributions of intent may be important in understanding why aggressive children encoded certain cues, there is no known way to measure hostile attributions as they occur in real-time.

This concept of real-time processing has also been studied in another SIP step in the literature. Fontaine and Dodge (2006) have developed a model of real-time processing that occurs at step 5 of the model, response evaluation and decision. Their model accounts for aspects of real-time decision making that they believe to be important such as working memory and impulsivity. Fontaine (2008) suggests that other factors such as nonconscious cognitions, emotion, and development should also be incorporated into models of real-time social decision making. Researchers' attention to real-time processing in social cognition has highlighted the need for and complexity of assessment of real-time social information-processing, which has been otherwise lacking in studies of the SIP model (Crick \& Dodge, 1994). Of specific interest in this study is how to apply real-time processing concepts to the study of step 2 of the SIP model, specifically attribution formation.

\section{Attribution Formation}

The social information-processing model suggests that, during its second step, children participate in an "interpretation of cues" and make causal attributions of another person's behavior in social interactions (Crick \& Dodge, 1994). "Causal attribution" refers to the thoughts that a child has, which identifies the actor responsible for an observed social stimulus (Weiner, 1985). In addition to causal attributions, attributions of intent occur within this step when a child takes the perspective of another person involved in the social event (Crick \& Dodge, 1994). Children who take the perspective of the other person may develop ideas about 
why a person acted in a certain way, or what their intent was. These intent attributions occur in the context of step 1 of the SIP model and help lead to the outcomes that ensue in steps 3-6.

Encoding and attribution formation. In order for cues to be interpreted, they must be perceived, which occurs in the first step of the SIP model, "encoding of cues" (Crick \& Dodge, 1994). The encoding step includes attending to and identifying social cues. Encoding implies that social cues are not just seen and heard but are also attended to, potentially in a selective manner. Once this occurs, it is believed that higher level brain processes identify the cues. Research has found that selecting which cues to attend to directly affects the formation of causal attributions (Lansford, Malone, Dodge, Crozier, Pettit, \& Bates, 2006). If cues are missed, attributions could be created without complete information, and therefore could be inaccurate. This could also occur if attention focuses on certain cues disproportionately, thereby assigning those cues greater significance than they actually deserve. It is assumed that social stimuli that are not within the realm of direct attention do not impact attribution formation or other steps.

New evidence using eye-tracking to measure encoding among aggressive children suggests that information-processing might not always occur in the "bottom-up" direction, with encoding leading to attributions (Horsley et al., 2010). Findings suggest that children exhibiting more aggressive behavior looked at non-hostile cues longer than children who did not exhibit aggression, possibly because they were expecting to see hostile cues. Authors suggest this could reflect a "top-down" approach whereby hostile attributions and schemas influence which cues are attended to (Horsley et al., 2010). More research is needed to determine the direction of the relation between encoding and hostile attribution, or to determine that the relation is bi- 
directional. This cannot be accomplished until real-time methods exist for understanding hostile attributions as they occur.

Causal attributions. Causal attribution formation is a cognitive process that incorporates information gathered from the social stimulus with a child's internal thoughts and beliefs. It serves to assign meaning and relevance to social stimuli by creating a personal perspective of the situation. The process of making causal attributions occurs as a function of one's internal cues and thought patterns, which can change from person to person and situation to situation. Several theories of attribution formation provide a framework for understanding how children make causal attributions.

Actor-observer effect. One attribution theory with empirical support purports that an actor in a social situation forms attributions differently than if that actor were just an observer of the situation. Research shows that people who observe social interactions make causal attributions that emphasize the role of those who are actively involved in the interaction (Gilbert \& Malone, 1995). Specifically, observers tend to attribute behavior to others' internal process or dispositional traits. This attribution is often an error because it fails to incorporate the role of external, situational factors. This bias in interpreting information in social situations has several labels, including the correspondence bias, the actor-observer effect, and the fundamental attribution error. In the context of hostile attributions of intent, we are interested in a person who is both actor and observer, in that they are actively involved in social interaction, but they are making an observation about their peer.

Theories about the actor-observer effect rely on several possible explanations for the phenomenon. For example, the bias seen in the actor-observer effect has been explained as a 
product of errors in encoding situational cues. Gilbert and Malone (1995) postulated that the correspondence bias resulted, in part, from a lack of awareness and perception of situational factors. They asserted that the observer needed to have knowledge about circumstances if he or she were to attribute behavior to them. Similarly, Malle, Knobe, and Nelson (2007) conducted research indicating that the differences between social cognitive processes in actors and those in observers related to their degree of knowledge. They noted that actors have more information, and therefore more knowledge, about their internal reasoning and beliefs than observers do. Observers are inherently missing important information about the actor and consequently about the situation because they are only observing. These theories of informational deficits suggest that attributional content relies on perception and awareness in real-time, which is reflected in the SIP model's explanation of how the encoding step leads to the interpretation, or attribution formation, step.

Additional research on the actor-observer effect has provided insight into the role that past experiences play in attribution formation. According to correspondence bias theory, as observers make attributions, they tap into beliefs and expectations about an actor's behavior. They may compare the information about the current situation with expectations they have developed based on past situations (Gilbert \& Malone, 1995) Relying on these expectations and beliefs can result in overgeneralized or unrealistic interpretations of the actor's role in a social situation. For example, research has shown that observers tend to emphasize the actor's personality if the observer has been involved in a similar social situation with the same person previously (Robins, Spranca, \& Mendelsohn, 1996). While this could lead to generalizations consistent with the actor-observer effect, additional evidence indicates that familiarity with another person could reduce the actor-observer asymmetry by increasing the amount of 
knowledge available to the observer (Malle, Knobe, \& Nelson, 2007). In other words, if an observer has a close relationship with the actor, the observer likely has increased information about the internal processes of the actor which is hypothesized to result in a weaker observer bias. Alternatively, if the observer does not have past experiences from which to judge the actor, the observer may exhibit a stronger bias in the direction of attributing outcomes to the actor.

Just as attributions are made using information from past social experiences, they may also be based on observers' schemas about their own relational style. These relational schemas are patterns of beliefs about how one relates to others across many situations (Baldwin, 1992). These schemas have been shown to be activated by social stimuli and used during real-time attribution formation (Lochman \& Lenhart, 1995). Internal schemas about one's relational style may be a reason that individuals seem to show consistent attributional styles, or a tendency to make certain types of attributions across a variety of types of social situations (Robins et al., 1996).

Stable attributional styles may also be explained by individual traits, which affect the way that people form attributions in response to social stimuli. Personality traits such as extraversion and impulsiveness result in different attributions of social cues. In the instance of extraversion, people with this personality trait were more likely to attribute their talkativeness to their own personality (Robins et al., 1996). If a person tends to be impulsive they are likely to choose to focus on the first or most salient cue in an impulsive manner rather than sort through the many social cues in each situation (Fontaine \& Dodge, 2006). While these traits may influence the attribution formation process, it is also possible that the process could also have a role in the 
development of traits. Early patterns in children's social information-processing may eventually develop into personality-like traits (Crick \& Dodge, 1994; Dodge, 2006).

\section{Hostile Attributions}

Crick and Dodge (1994) hypothesized that attributing social circumstances to others' hostility would be an impetus for developing and enacting aggressive responses. There are many reasons that one might make a hostile attribution of intent about another person. The most common and adaptive way of forming a hostile attribution of intent occurs when one accurately identifies the malicious intent of another person by encoding social cues. These cues can be overt such as threatening language or aggressive posturing. Cues implying hostile intent might also rely on more covert methods such as sarcasm or deception. As mentioned earlier, attribution formation also relies on encoding of one's internal cues such as sympathetic nervous system arousal or anger. These can occur as part of a correct assessment of another's intentions, or they can contribute to a hostile attribution of intent that is not accurate. Traditionally, this "bottom-up" explanation of social information-processing argued that hostile attribution biases result from errors in the first step of the SIP model, encoding, or during the interpretation step (step 2; Crick \& Dodge, 1994). Alternatively, it has been suggested that these encoding and attribution errors could result from "hostile intent" schemas being activated and used to make interpretations and to choose which cues to encode (Horsley, et al., 1995).

Research has clearly found that one's past experience plays a key role in how children interpret social situations (Lochman \& Dodge, 1998; Steinberg \& Dodge, 1983). These past experiences form children's mental representations of social situations in internal schemas. When similar social situations recur, memories are triggered in schemas, which then provide a 
framework for interpretation of the situation. For example, aggressive children appear to have expectations that they will, again, react aggressively (Lochman \& Dodge, 1998). In the case of hostile attribution of intent, repeated exposure to hostile intentions could lead to the negative encoding and interpretation of a number of social cues. Indeed, studies show that children whose parents exhibit hostile parenting behavior tend to make hostile attributions (MacKinnon-Lewis, Lamb, Hattie, \& Baradaran, 2001). Another approach to understanding hostile attribution of intent suggests hostile attributions exist as humans' default attribution before we ever experience interaction with others.

Dodge (2006) suggests that hostile attribution is a default attributional style that children are born with due to evolution of brain systems. He cites evidence that hostility is a lower level brain function that was useful for primitive species but that is usually maladaptive for modern humans. According to Dodge, children ordinarily learn that hostile attributions are not always accurate or adaptive as they experience various social interactions. Hostile attributions are replaced with a wider set of causal attributions to consider, which is regarded as more adaptive. Some children may not be presented with ample opportunities to learn that people sometimes have non-hostile intentions. If children are surrounded by violent or contentious environments, their default hostile attribution may be reinforced rather than challenged. According to Dodge (2006), children who do not develop a repertoire of causal attribution options may exhibit a propensity to rely on attributions of hostile intent to explain others' behavior.

\section{Hostile Attributions and Emotion}

Hostile attributions are associated with the perception of a threat or hostility and it has been theorized that such a perception is associated with emotional arousal. Crick and Dodge's (1994) social information-processing model made mention of emotional arousal, but did not 
cohesively incorporate emotion into the model. As emotion relates to step 2 (interpretation), the authors suggested that the interpretation of hostility could lead to fear or anger, which could further result in preemptive processing, rather than deliberative information-processing. More recent research has suggested that emotion should be an integrated part of the social informationprocessing model because children can come into a social interaction in pre-existing emotional states or with schemas that include affective components from past interactions, in addition to the emotion generated within the social interaction itself (Lemerise \& Arsenio, 2000). The emotional cues of a peer in a social situation, as well as schemas, might be particularly influential on the attribution step.

Evidence supports various roles of emotion in the development of hostile attribution. Research suggests that dispositional anger moderates the relationship between hostile attributions and aggression, with hostile attributions showing the most association with aggression when a child also tends to show dispositional anger (Runions \& Keating, 2010). This relation appears to exist not just when anger is measured as a trait, but also when emotion is measured as a state. Among aggressive children, more hostile attributions tend to be made when a negative emotional state was induced, as opposed to a positive emotional state (Dodge \& Somberg, 1987; Orobio de Castro, Slot, Bosch, Koops, \& Veerman, 2003). Emotion was measured in terms of arousal reactivity by Williams, Lochman, Phillips, and Barry (2003) who found that moderately aggressive boys exhibited increased heart rate and increased hostile attributions when a threat was introduced. In a test of Lemerise and Arsenio's (2000) theoretical model including emotion, hostile attributions, and aggression, boys' report of their own anger was related positively to their hostile attributions, as well as to their aggression (Orobio de Castro, Merk, Koops, Veerman, \& Bosch, 2005). In this same study, boys' interpretation of a peer as appearing angry was related 
to increased hostile attributions. This suggests that internal and external anger cues are important as children make hostile attributions.

\section{Attributions and Behavior}

As is postulated in the SIP model, attributions are thought to be one of the cognitions contributing to a specific behavior. While the SIP model illustrates how a number of perceptions and cognitions can lead to a single act such as aggression, other models provide ideas about the link between cognitions and behavior. On a broad scale, information-processing theories have often emphasized the importance of cognitions in the determination of behavioral responses. The theory of reasoned action (Ajzen \& Fishbein, 1980) and its subsequent revised version, the theory of planned behavior (Ajzen, 1991; Armitage \& Conner, 2001), suggest that one's beliefs contribute to cognitions, which result in an intention to enact a behavior followed by the actual enactment. Other theories de-emphasize the reasoning and planning of these theories and focus on behaviors stemming from impulsivity and automaticity (Ouelette \& Wood, 1998; Strack \& Deutsch, 2004).

In the specific realm of attribution formation, Weiner (1985) asserted that causal attributions predict how one later feels about a social stimulus as well as how one responds to it. Attributions are linked to later steps in the SIP model as well, because interpretation of cues (step 2) aids in clarifying one's individual goals (step 3), which then leads to choosing and enacting a behavioral response (steps 4-6) (Crick \& Dodge, 1994). Identifying the actor and other potential causal factors in a social situation is essential for the reasoned problem solving and decision making, which occur following attribution formation. Because attributions influence the reasoning of and response to social stimuli, mistakes, omissions, or distortions in attribution 
formation can lead to negative social behavior. The attribution style that has been consistently linked to aggressive behavior is hostile attribution of intent.

\section{Hostile Attribution Bias and Aggression}

Attributing social circumstances to others' hostility, or forming a hostile attribution, is hypothesized to be an impetus for developing and enacting retaliatory responses (Crick \& Dodge, 1994; Dodge et al., 1997). Hostile attribution has also been conceptualized, not just as a momentary cognition, but also as a cognitive style that persists across a variety of contexts (Dodge, 2006; Dodge \& Pettit, 2003). Children sometimes show a consistent proclivity to interpret ambiguous provocation as intentionally hostile. Children who have these cognitions repeatedly make the assumption that they are being treated with hostility, or attacked, even with a lack of observable hostile cues (Dodge, 2006). This results in a hostile attribution bias. Moreover, it is believed that children use this perception when formulating their behavioral responses, as explained above. Children making hostile attributions about the actions of others are thought to be more likely to formulate a corresponding reactive response - specifically, reactive aggression (Dodge et al., 1997; Orobio de Castro et al., 2002).

While theory has predicted that hostile attribution bias would be associated with aggressive behavior (Crick \& Dodge, 1994), research has supported a moderate relation between hostile attribution of intent and aggression. Orobio de Castro and colleagues' (2002) review of 41 studies of the attribution to aggression link found a small overall effect size $(r=.17)$, but average effect sizes varied from small to large depending on the method used to measure hostile attributions. Average effect size was largest $(r=.55)$ among those studies that used staged social interactions to measure hostile attribution. Effect sizes in studies using primarily visual stimuli, 
including pictures and videos, was low $(r=.02-.09)$. Studies using hypothetical vignettes as a stimuli showed a moderate effect size $(r=.24)$. Research also shows that hostile attribution predicts aggressive behavior across time, which supports the SIP model's serial pathways beginning at cognition and leading to behavior (Lansford et al., 2006).

Reactive vs. proactive aggression. A specific subtype of aggression, reactive aggression, has been investigated as a strong correlate with hostile attributions. Reactive aggression has been described as an emotional and poorly-controlled aggressive response to a provocation or to frustration (Dodge \& Coie, 1987). Unlike proactive or instrumental aggression, reactive aggression occurs primarily as a reaction to a social stimulus, often in an attempt to defend oneself from a perceived threat. Reactive aggression can result from associated feelings of frustration and anger (Berkowitz, 1989). Proactive or instrumental aggression has been characterized as controlled, deliberate, and goal-driven aggressive behavior (Dodge \& Coie, 1987). Unlike reactive aggression, proactive aggression is likely to be rational and unemotional and result in specific rewards, which reinforce the aggression (Bandura, 1973).

Because reactive aggression theoretically stems from one's interpretation of his or her environment, it has been predicted to be more strongly linked to maladaptive interpretations such as hostile attributions. As would be expected, reactive aggression, more than proactive aggression, has been empirically linked to hostile attribution bias (e.g., Arsenio, Adams, \& Gold, 2009; Bailey \& Ostrov, 2008; Crick \& Dodge, 1996; Dodge \& Coie, 1987; Dodge et al., 1997). This finding has been exhibited via several methods. In an observational study, hostile attribution has been linked to observed reactive aggression responses (Hubbard, Dodge, Cillessen, Coie, \& Schwartz, 2001). Children who made hostile attributions about ambiguous 
provocations were more likely to respond to those provocations with reactive aggression. In correlational studies, a history of reactive aggression has been related to a tendency to make hostile attributions. Children who have been identified by parents or teachers as exhibiting high levels of reactive aggression have also exhibited high levels of hostile attributions (Dodge \& Frame, 1982; Dodge \& Coie, 1987; Nas, Orobio de Castro, \& Koops, 2005). The relation between hostile attributions and reactive aggression has been found to be similar across cultures and genders (Crick \& Dodge, 1996; Xu \& Zhang, 2008). The effect has also been shown in samples ranging in ages from school-aged children (e.g. Crick \& Dodge, 1996; Dodge \& Coie, 1987) to emerging adults (Bailey \& Ostrov, 2008).

Though reactive aggression is accepted as a correlate of hostile attribution bias, literature does not universally rule out proactive aggression as another potential correlate, albeit a weaker one. In Orobio de Castro and colleagues' meta-analysis (2002), the exclusion of proactively aggressive children resulted in a lower average effect size of the hostile attribution and aggression relation, indicating that proactive aggression could have an additive effect on the relation between hostile attribution bias and reactive aggression. The relations between hostile attributions and proactive versus reactive aggression are complicated by the positive correlation found between proactive and reactive aggression. Research shows that the two forms of aggression are closely linked, but that they often relate differentially to individual risk factors, such as hostile attributions (Card \& Little, 2006; Poulin \& Boivin, 2000). Researchers have managed this apparent contradiction by using partial correlations to test the effect of each form of aggression, after partialling out the variability accounted for by the other. As an example, Orobio de Castro and colleagues (2005) found hostile attribution to be related to reactive and proactive aggression using zero-order correlations, and to be related to reactive, but not 
proactive, aggression when using partial correlations. While reactive aggression is consistently found to be a correlate of hostile attribution bias, additional research is needed to determine the nature of the relation between hostile attribution bias and proactive aggression.

Age as a moderator. The relation between hostile attributions and aggression appears to be moderated by the age of the child. Orobio de Castro and colleagues (2002) found that studies of children ages 8 to 12 years old had larger effect sizes than those of children ages 4 to 6 , ages 6 to 12 , or ages 12 and over. The authors suggested that it was unclear why this "wave-like pattern" of the magnitude of the effect size occurred across ages. Some additional evidence suggests that the age range between 9 and 12 has been linked to a number of variables including decreases in parental monitoring and increases in the hostility of the school environment, child autonomy within the school environment, and peer influence on children (Blyth et al., 1978; Eccles et al., 1993; Fite et al., 2006). Research has indicated that the role of peer influence on children also shows a curvilinear pattern in which children's conformity with their peers peaks during the middle-school years (Berndt, 1979). When taken together, the unique changes of this developmental age, along with the findings of Orobio de Castro and colleagues (2002), suggest a need for additional research on the way that hostile attribution and aggression relate in this preadolescent age group.

\section{Hostile Attribution Measurement}

A variety of measures have been employed to measure hostile attributions, though each captures the construct by first presenting the participant with an ambiguous social interaction and then eliciting his or her interpretations of that interaction. Presenting a research participant with an ambiguous social stimulus has taken many forms, including written accounts, videos, and live 
experiences of social interactions. The stimuli characteristics appear to be important because different stimuli result in different magnitudes of effect sizes in studies of hostile attribution's ability to predict aggression (Orobio de Castro et al., 2002). Additional variation in attribution measurement occurs in the way that responses to stimuli are elicited and evaluated, which has included multiple choice response options and open-ended questions.

Stimuli characteristics. Many types of stimuli have been used to simulate a social situation with an ambiguous provocation in the experimental setting. Visual stimuli have included videos, drawings, pictures and cartoons depicting social interactions (Leff et al., 2006; Lochman \& Dodge, 1994; Weiss, Dodge, Bates, \& Pettit, 1992). In these media, short social interactions, or vignettes, are presented visually to the participant. These vignettes can vary from simple depictions of ambiguous provocation to detailed social interactions that include many pieces of social information, as well as an ambiguous provocation. In most cases, if real people are seen in the videos or pictures, they are confederates acting out a sufficiently ambiguous social situation. Some measures ask the participants to imagine that they are a child in the scene while others simply ask the participating children about the picture itself (Leff et al., 2006).

Some of the cartoon-based measures have coupled visual stimuli with a narrative story read aloud to participants that describes what is happening in the depiction (Leff et al., 2006; Weiss et al., 1992). Narrative stimuli have also been used alone as auditory stimuli. These scenarios usually consist of a social interaction being read either on an audiotape or aloud in the room with the child (e.g. Dodge \& Frame, 1982; Lochman \& Dodge, 1994). As with the visual stimuli, these auditory vignettes vary in the amount of detail and social information they include. Some measures ask children to imagine that they are actively involved in the scenario and others 
ask children to act as an observer of the people in the scenario, which could account for differences in findings.

Another method of presenting a social scene in a hostile attribution measure requires the participant to be directly involved in a live social interaction. "Staged situation" stimuli engage children in an actual ambiguous scenario, which is controlled in the laboratory setting. In Steinberg and Dodge's (1983) staged situation, children were told that they would be competing against a same-aged peer in a block-building contest. After building a tower and subsequently meeting the competing peer, the participant saw that his structure had been knocked over. Next to the fallen structure, the peer was playing with his own set of blocks. In this example, a carefully orchestrated series of events with a confederate peer resulted in a real-time experience of a social interaction. The participant observed his peer, but did not speak with him which helped to ensure consistency of the social stimulus across all participants. The participant was not given any clues as to how his structure came to be knocked over. The ambiguity and provocation inherent in this example make it a realistic stimulus for testing hostile attributions, though it is the only published test of hostile attributions using staged situations known to this author.

Measurement stimulus as a moderator. Different stimuli for measuring hostile attributions (e.g. staging an interaction, watching a video, reading hypothetical vignettes) are associated with varying effect sizes of the relation between hostile attribution bias and aggression (Orobio de Castro et al., 2002). Most measurement of hostile attribution bias has relied on narrative stimuli involving presenting a story or scenario to a child verbally and asking for his or her interpretation of the event using open-ended questions (e.g. Lochman \& Dodge, 
1994) or questions with multiple choice answers. Studies that used these traditional methods of measuring hostile attribution biases to predict aggression averaged effect sizes between $r=.18$ and .44 (Orobio de Castro et al., 2002). Another method of measuring hostile attribution bias relies on videos or pictures to portray scenarios that children are then asked about (e.g. Dodge \& Coie, 1987). Studies using these visual stimuli found that hostile attribution bias and aggression had a small relation with average effect sizes between $r=.02$ and .09 (Orobio de Castro et al., 2002). The type of stimulus that has been most successful in detecting an effect between the two is staging an actual interaction which has produced hostile attribution to aggression effect sizes averaging $r=.55$ (Orobio de Castro et al., 2002). The differences in effect sizes of the relation between aggression and hostile attribution depending on the method used for measurement suggest that these methods are qualitatively or functionally different from one another.

Response option characteristics. Across the different types of stimuli in hostile attribution measurement, various manners of eliciting interpretive responses are used. A common response format is multiple choice which provides the participant with prototypical responses about the intent of the actor in the stimulus. For example, the choices accompanying a set of commonly-used, narrative vignettes indicate that the provocateur did the provocation on purpose, the provocation was an accident, or the participant does not know why provocation occurred. By providing options, multiple choice responses necessarily limit the possible responses a child can provide. This is not a good proxy of internal processing, which can be quite complex. Multiple choice responses also reveal to the child the experimenter's goal of determining whether or not a hostile attribution was made. This could lead respondents to attempt to answer in socially desirable ways. 
Another response format has allowed participants to answer open-ended questions about the scenario. For example, the child may be asked why the peer might have done what they did (Weiss et al., 1992). In some studies, additional probing questions may be asked such as "Do you think he did it on purpose?" (Steinberg \& Dodge, 1983). Other open-ended response formats, such as the verbal protocol used by Milch-Reich and colleagues (1999), simply state that the participant should tell them what is happening. In most measures that use open-ended response formats, coding dichotomizes responses into hostile attribution of intent or non-hostile attribution of intent (Crick, 1995; Weiss et al., 1992).

\section{Problems with Hostile Attribution Measurement}

As discussed above, a number of types of stimuli and response options have been used in the measurement of hostile attributions. It was also noted that these differences in hostile attribution measures contribute to differences in the magnitude of the relation found between hostile attribution bias and aggressive behavior. In particular, staged situations were associated with the largest effect sizes, which could be explained by the level of engagement required in staged situations. Several characteristics of engagement in measurement instruments are hypothesized to be the most important in creating a valid measure of hostile attributions.

Personal involvement. While each measure described above purports to examine the unified construct of hostile attribution, some stimuli and response options appear to improve the validity of those measures. One measurement validity issue centers on an instrument's ecological validity, including the ability to reproduce a real hostile attribution in a research setting. Specifically, Orobio de Castro and colleagues (2002) suggested that the participant's perception of personal involvement was reduced when using video clips and reading hypothetical vignettes. Indeed, the authors asserted that a reduction in personal involvement of the participant 
accounted for lower average effect sizes found among hostile attribution measures that used these video and audio stimuli. Alternatively, staged situations that directly involved the participant greatly increased their level of personal participation, which could have affected the level of hostile attributions that they reported (Orobio de Castro et al., 2002).

In support of this idea, research has shown that attributions made by active observers differ in content and frequency from those made by passive observers. Participants, who are actively engaged in an interaction, or active observers, attribute the behaviors of the person with whom they are interacting to that person (Miller \& Norman, 1975). In contrast, when people are observing a situation that they are not actively involved in, they are more likely to attribute people's behaviors to the situation (Miller \& Norman, 1975). The differential effect of active versus passive observer on attribution formation has been replicated in the study of hostile attribution and aggression in children. Dodge and Frame (1982) showed that aggressive boys did not make more hostile attributions than nonaggressive boys when they were passive observers, but did when they were actively involved in a situation. In other words, children's personal involvement moderated the hostile attribution and aggression relation. It then follows that measurements of hostile attributions should directly involve participants to most closely approximate real-world situations.

To improve personal involvement, measures of hostile attributions should also allow personal responses. Multiple choice response options do not permit children to explain their own thoughts and reasoning, even though that is a primary goal of the measure. Open-ended and stream-of-consciousness responses allow a child to be more involved in responding and selfreflective in his or her answer. 
Real-time processing. Though real-time processing in the interpretation stage of the SIP model has been identified as an important area to be studied (Crick \& Dodge, 1994), measurement of on-line attribution formation has been limited in the past. Research on other steps within the SIP model such as response evaluation and decision making has suggested that measurement of real-time processing could include virtual reality experiences of social interaction, real social games, or staged interpersonal exchanges (Fontaine \& Dodge, 2006). As yet, few of these techniques have been attempted in the study of hostile attributions (Orobio de Castro et al., 2002). As described above, in Steinberg and Dodge's (1983) staged situation, children experienced a social situation by observing that their structure had been ruined presumably by a peer. While techniques such as this allow real-time provocation, hostile attribution processes are not measured until after the event. In real life, hostile attributions are likely to occur instantaneously. In order to focus hostile attribution measurement on real-time processes, responses to a staged social interaction should be elicited immediately after, or even during, the interaction.

\section{Proposed Method for Measuring Real-Time Hostile Attributions}

As discussed above, measures that optimize a participant's personal involvement in the social stimuli and those that measure real-time processing may be better at replicating real-world hostile attribution formation, and its relation to aggressive behavior. In a research setting, a social stimulus that engages a child in an actual social interaction should ensure high personal involvement in several ways. First, the stimulus should require that the participant actively engage in an activity. Ideally, this activity could feel socially interactive to the child. In order for this to occur the child should interact with a peer or perceive that he or she is interacting with a peer. Additionally, the interaction should involve some ambiguous provocation by the peer 
that is standardized across participants. When these conditions are met, measurement of hostile attributions should closely mirror what a child's hostile attribution would be in a similar real-life situation.

Video gaming. Some previous research on children's social interactions has used video games to approximate a social interchange without using an actual peer. Instead, these studies use an unseen "opponent" as an assumed peer, though the opponent is actually being manipulated by the experimenter. For example, Dorsch and Keane (1994) used a computer game to experimentally manipulate children's engagement in a cooperative or competitive interaction. They identified computer games as an "ecologically valid" method of studying children's social information-processing (Dorsch \& Keane, 1994). They noted that engaging children in playing a computer game would produce "realistic personal involvement" (Dorsch \& Keane, 1994).

Several forms of computer games have been used in research on children's aggressive behavior. Dorsch and Keane (1994) used a math game against a fictional peer opponent which was designed to defeat the participant in half of the games and to be defeated by the participant in the other half of games. In this study, hostile attributions were measured using a traditional vignette measure after the video games were completed. A recent study of girls ages 9 to 12 used a computerized board game and chat room in which participants could send messages to a fictional peer about the game (Ohan \& Johnston, 2007). Researchers found that the game and subsequent "chats" were able to elicit overt aggression and relational aggression, though no measure of hostile attribution was used in that study. Several other studies have used a pinball computer game in which the fictional peer opponent and the participant can tilt the game table or provide a burst of white noise to annoy each other (Atkins, Stoff, Osborne, \& Brown, 1993; 
Phillips \& Lochman, 2003). This pinball game method has been shown to be an effective analog for aggressive behavior.

Given the success of using video games in previous research of aggression and social information-processing, computer games appear to be an ecologically valid tool for measuring real-time hostile attributions. As described above, using a computer game involving a fictional peer would ensure that the participant is personally involved in the social interaction. It would also allow for real-time responses to be collected while the child is playing the game. Just as previous research has allowed for real-time aggression to occur in computer games (Phillips \& Lochman, 2003; Ohan \& Johnston, 2007), a computer game could also be used to elicit real-time hostile attributions.

Verbal response data. To assess real-time attribution formation, measures of hostile attribution should include immediate response to the stimuli. In order to promote the most online response, participants should be encouraged to give their interpretation of a social event as the interaction occurs. The interpretation could take the form of a verbal explanation of the scenario or could include nonverbal communication of one's interpretation. Verbal explanations, such as verbal protocols, allow a participant to speak freely while experiencing the stimuli without being influenced by the suggestions of potential attributions by the experimenter (Ericsson \& Simon, 1980). In the context of an information-processing model, Ericsson and Simon (1980) postulated that verbal protocols could be used to tap information that is being attended to in one's short-term memory. Verbal protocol analysis is the process of reviewing a transcript or tape of the verbal protocol that allows a researcher to determine what the participant has attended to and how they have interpreted that information (Ericsson \& Simon, 1980). In 
this way, verbal protocol analysis allows researchers to measure and examine real-time cognitions.

Verbal protocol analyses, as well as the related technique of coding narrative responses to open-ended questions, have been used in some studies of children's social informationprocessing. Milch-Reich and colleagues (1999) used this technique to measure real-time encoding of social cues in a depiction of a social interaction. Another study asked children to draw a bullying interaction and used open-ended questions about the drawing to allow children to develop a narrative of what happened in the bullying picture (Bosacki, Marini, \& Dane, 2006). Additionally, a number of measures of hostile attributions using vignettes have used open-ended questions. Responses are then coded for the presence of hostile attributions.

Using verbal protocol analysis to measure real-time hostile attribution bias has some potential advantages, as well as several limitations. Because verbal measures of attribution formation require language production, they could be difficult for children with poor expressive language skills (Milch-Reich et al., 1999). On the other hand, the verbal protocol technique does not suggest any restraints or demands on what language is produced, permitting children to respond using whatever language skills they have. Milch-Reich and colleagues (1999) used a very sensitive coding system to account for their respondents' potential language difficulties. In this example, even several words indicating the beginning of attributions were counted as complete attribution of intent statements. Still, children who fail to express an attribution of a peer's intent in a verbal protocol may do so because they never formed an attribution. To help decipher whether children are failing to make hostile attributions or whether they have trouble verbalizing their hostile attributions, nonverbal methods of measuring hostile attribution could also be used. 
Nonverbal response data. While some children may have difficulty expressing their interpretations verbally, nonverbal response techniques have been shown to be effective in gathering data in a range of ages and levels of functioning. Nonverbal, real-time measures have been used to gauge respondents' interpretations across the social sciences. For example, dials turned by research participants have been used to rate interpretations of politicians' speeches and commercials (Alwitt, 2002), feelings while listening to music (Parisi, 2004), and reactions to parenting behavior (Lorber, 2007). These dials have been used with children as young as second graders (Frederickson, 1997). Another study required second and fourth graders to press a button when they observed a child changing tasks in a video (Graziano, Moore, \& Collins, 1988). These findings seem to indicate that children are capable of providing a nonverbal response while they are interpreting a social situation. In order to maximize both on-line processing measurement and personal involvement with the stimulus, nonverbal and verbal feedback should both be gathered while the participant is engaged in a staged situation.

\section{Hypotheses}

The study investigated the validity and reliability of several new ways of measuring hostile attribution biases. In particular, the measurement methods attempted to reflect real-time processing that occurs in children's everyday lives. These real-time hostile attributions were measured using a verbal and nonverbal response procedure during children's interaction in a staged social situation, which involved response prompts at ambiguous provocations (crashes) and non-provocations (non-crashes). In order to determine the usefulness of these new ways of measuring hostile attributions, a more traditional measure of hostile attributions, based on hypothetical vignettes, was also administered to participants. Because the goal of measuring 
hostile attributions is to understand children's aggressive behavior, aggression was measured by parents' ratings in the areas of proactive aggression and reactive aggression.

Hypothesis 1. The primary hypothesis of the current study is that the percentages of both verbal and nonverbal real-time attributions that are hostile will significantly relate to aggressive behavior. The percentage of hostile attributions made in response to crash provocations will also be related to aggression. Because hostile attributions are expected to spill over between crashes, the percentage of attributions made in response to non-crashes is also expected to relate significantly to aggression.

Hypothesis 2. The study measured different types of aggression as outcome variables. The verbal and nonverbal measures of real-time hostile attribution are expected to significantly relate to reactive aggression after controlling for proactive aggression. They are not expected to relate to proactive aggression, after controlling for reactive aggression.

Hypothesis 3. It is expected that the combination of verbal and nonverbal measures will significantly predict reactive aggression, but not proactive aggression. This combination of verbal and nonverbal measures is expected to predict reactive aggression, when controlling for proactive aggression, better than either measure alone.

Hypothesis 4. The new real-time measures were expected to be better predictors of children's reactive aggression, after controlling for proactive aggression, than traditional, vignette-based measures of children's hostile attributions. 


\section{CHAPTER 2}

\section{METHOD}

\section{Sample}

The sample included 75 girls $(n=22,29 \%)$ and boys $(n=53,71 \%)$ ages 10 through 13 $(M=11.84, S D=.74)$. The sample consisted of primarily African-American youth $(n=60$, $80 \%)$, with smaller groups of Caucasian youth $(n=12,16 \%)$ and youth from multiple ethnicities $(n=3,4 \%)$. Participants attended public school in Tuscaloosa City, Tuscaloosa County, or Bessemer City school districts of Alabama. The participants comprising the sample were recruited from participants of two larger studies of a preventive intervention for aggression in children. One of the larger studies consisted of 120 participants, while the other study consisted of two cohorts of 120 participants each. As part of the larger studies, children's parents were asked if they would be interested in being contacted for future studies, and, if so, signed a consent-to-contact form. These families were then contacted by phone and the procedure of the current study was explained to them in order to determine their interest in participating.

Participants who were included in the larger studies were initially identified as at-risk for developing aggressive behavior. Children's aggressive behavior was rated by their classroom teacher using a short screening measure. Those participants whose aggression scores on the measure fell into the top $25 \%$ of students were recruited. The resulting sample, from which the 
current study drew participants, consisted of children identified as "at-risk" for aggressive behavior.

The "at-risk" sample is thought to provide benefit over a random sample of children of the same age because the "at-risk" sample likely provides more variability among aggression ratings in moderate to high levels of aggression. This variability at higher levels of aggression is important to detect the effect hostile attribution has on aggression. In other words, a non-risk sample would include a number of children who exhibit no aggression, and therefore who could not demonstrate the predictive validity of measures of hostile attribution. The "at-risk" sample also contributes to the external validity of the study. The primary implication of this study is better measurement of hostile attributions and better understanding of the relation between hostile attribution bias and aggressive behavior. Because the "at-risk" sample allows examination of these individual differences in the attribution-aggression link, it offers an opportunity to aid in the development of tailored interventions based on these individual differences.

\section{Procedure}

The current study was preceded by a pilot study of six children to examine the feasibility of the procedure for the larger study. Lessons learned from the pilot study were incorporated into the procedure described below. Specifically, it was noted that the previous instructions were not explicit enough for some children. The current procedure reflects an expanded practice session in which the investigator interacts with the participant to ensure that he or she

understands the response procedure prior to beginning the races. Additionally, some spillover of emotion and attribution was thought to be possible between the two races. A distraction task, in 
the form of watching a calming video of scenic Hawaii, was added between the two races. Finally, some pilot participants expressed feeling tricked at debriefing. As a result, changes were made to the debriefing procedure to include questions leading participants to discover the deception in a more gradual way.

Participants completed the study either in the research office or in their homes. While the first 16 participants were asked to come into the research office, this method resulted in a number of participants missing their appointments due to difficulty with transportation or the inconvenience of having to travel to The University. As a result, subsequent participants were given the option of completing the study at The University or at their home. Following the initial 16 participants, twelve more families came to the research office to complete the study, resulting in 28 participants (37\%) engaging in the study in this setting. The remaining 46 participants $(61 \%)$ completed the study in their home. One participant requested that the study be completed at a local fast food restaurant. When participants completed the study in the research office, parents and children were separated and most parents did not observe their child participating in the real-time measure of hostile attribution, though many were curious to see the computer game when the study ended. At participants' homes, efforts were made to allow the child to participate in a separate area from his or her parent, though this was not always possible.

The procedure consisted of a parent informed consent and child assent process. Parents were given self-report measures to complete, while their child engaged in the task. Youth were asked to respond verbally to a traditional vignette-based measure of hostile attribution bias. Their responses were audio-recorded in most cases and coded later. Children then completed the real-time measure of hostile attribution that was designed for this study. Finally, they answered 
several questions about their video game use and participated in a debriefing process with their parent.

Real-time measurement of hostile attributions. In order to simulate real interactions with peers, participants used a gaming steering wheel connected to a computer to manipulate a racecar in an interactive racing game, which was created for this study (Loewald, 2009). Participants completed a brief practice period, followed by two 5-minute races against an opposing car. The racing game screen shows a horizontal split screen with the opponent's car on the top portion of the screen and the participant's car on the bottom portion of the screen. The two cars are on the same video game track, which is the shape of a large figure- 8 with city scenery surrounding it.

The racing game allowed a social interaction to be staged, as the participant played the game with an opposing racecar. Participants were told that the operator of the opposing car was a peer of his or her same age and gender during the first race. During the second race, they were told that they would be racing a different peer of his or her same age and gender. This was done to remove any spillover effects from Race 1 to Race 2 . In both cases, participants were informed that they would not see or hear the opponent, and would not meet after the race. Participants were told their opponent could see the same images on the screen that they did. The opposing car was actually operated by the computer software, which allowed the interactions in the game to be standardized across participants.

The behavior of the opposing car was programmed to include ambiguous provocations in the form of crashes. Their role in the racing task was to elicit interpretive cognitions, or attribution formation, in the participants. As in traditional, vignette-based hostile attribution 
measures, the provocation needed to be ambiguous to enable interpretation as hostile or nonhostile. In order to achieve this, participants were told that their car was on the same team as the opposing car, and that their team was racing against another team. The participant's car was always red, while the opponent's car was always yellow. Two other cars, blue and green, were identified as the other team. The child was told that he or she was also competing against the opposing car to win the race, as there can only be one winner. In this way, the current racing task was framed as both a cooperative and competitive endeavor.

Children completed an instruction period during which they had the opportunity to practice the verbal and nonverbal response procedures described below. The instruction period lasted several minutes or until participants demonstrated an understanding of the response procedures. Participants then had the opportunity to play the game independently for several additional minutes to become acquainted with the controls of the game. During that time, the yellow car did not appear in the upper portion of the screen, so that the participant could focus on practicing with his or her own car. Following that, participants played the game with the opposing car for two identical race sessions of about 5 minutes each. Participants were asked to use the nonverbal response procedure during one race and the verbal response procedure during the other race. The order of the races was counterbalanced such that half of the sample completed the nonverbal measure of hostile attribution during the first race and the verbal measure during the second race. The other half of the sample completed the verbal response procedure during the first race followed by the nonverbal response during the second race.

In both races, participants experienced ambiguous provocation when opponents' cars crashed into their car at pre-programmed intervals throughout the race. In addition to the ambiguity provided by the cooperative and competitive nature of the task, the crashes of the 
opposing car were designed to be unclear in their intent. The game used a text document containing input commands that dictated when the program created crashes. The software is designed to bring the opponent's car to within striking distance behind the participant's car, wherever it is on the race track, and proceed to crash into it at the designated crash moment. The result was a variety of types of crashes including those from the sides and from the rear. Some crashes resulted in a complete stop of both cars, other produced cars flipping and spinning around, while others were side-swipes that just bumped the cars without stopping them or even missed direct contact.

A total of eight provocations, or crashes, occurred during each of the two 5-minute races. A random digit generator was used to determine at which second during the 300-second (5minute) races provocations would occur. At each instance of provocation, participants were prompted to provide a verbal or nonverbal interpretation as the measure of hostile attribution bias. The result was eight "items" or instances of provocation and prompted responses. Eight additional event times were randomly generated to prompt for a response without a crash occurring. These eight non-crash prompts served as a control, and were interspersed with the eight provocation prompts during the 5-minute race. The result was two identical races, each 5 minutes long, with one using the nonverbal response procedure and the other using the verbal response procedure. During each race prompts appeared 16 times, with eight crash prompts occurring immediately after provocations and eight non-crash prompts occurring without provocation.

Debriefing. Several previous studies using fictional peers to simulate social interaction have described important points in their debriefing processes. For example, one study explained the purpose of the study to participants during the debriefing process and why deception was 
necessary to achieve that purpose (Ohan \& Johnston, 2007). Hubbard (2005) outlined an ethical procedure for provoking children in research settings. Her procedure included fully informing parents about the procedures prior to their children's participation. The debriefing process used in this procedure also emphasized that the participant was helping the researcher by completing the study and that the participant did not lose the game because he or she was a bad player (Hubbard, 2005). The debriefing process for the current study included these elements as well and is described below.

While participants' parents were told that their child was racing against a computeroperated car, participants were told that the opposing car was a same-aged peer. Debriefing regarding this deception occurred at the end of the study. Participants' parents were present when the investigator explained that the opposing car was operated by the software rather than an actual peer. In order to determine if the parent had any concerns about the deception, they completed a debriefing questionnaire (see Appendix), which the investigator reviewed with them prior to debriefing. Several parents did express concern that their child might feel tricked, though none thought this would be a problem or felt as though they wish they had not participated. In cases where parents had qualms about the deception, they were discussed prior to debriefing and parents stated their fears were allayed in every case. In two cases, the investigator called the participant's parent several days after the study to determine if there were lingering effects of participation. The parents reported that they had no further questions or concerns.

To begin the debriefing process, questions were asked of the participant to determine if he or she had any suspicion that the game had been played against a computer-operated opponent rather than a real peer. Participants were asked "Was there anything strange or weird about the 
game?" and "Did the other player seem to play like a real 10/11/12/13 year-old would play?", and "Did it seem like there were a lot of crashes?" If respondents said yes, they were asked to elaborate. Few participants indicated having any idea that they might not have been playing against their peer.

During debriefing, it was explained to participants that there were many crashes because the study was designed to find out what children think about other children crashing into them during a racing game. They were told that the crashes were pre-programmed. It was explained that, in order to prevent a real child from having to crash into them, they were playing against a computerized car. Participants were reassured that they did a very good job considering how hard the game had been. The debriefing procedure also stressed that the participant's completion of the study would help us learn about how children respond to this type of difficult situation. Children also had the opportunity to pick a small prize from a prize box to thank them for participating. Parents and children had the opportunity to discuss any concerns about the study, though none were voiced following debriefing.

\section{Measures}

Nonverbal measure of real-time hostile attribution bias. In order to measure hostile attribution bias in real time, participants used a button on their steering wheel controller to indicate their perception of their peer's intent as they played the game. In order to elicit this response during incidences of provocation, a graphic prompt depicting a neutral face was displayed automatically on the screen to indicate that the participant should respond. This indicator, or response cue, was located in the upper portion of the screen, next to the opponent's 
car. Participants were instructed to press a response button when the response cue was on. The cue remained on for 5 seconds to allow participants time to shift their attention to the cue.

The participants pressed a left or right button on their steering wheel to indicate their peer's intent. Participants were instructed that the right button should be used to indicate that their peer is intending to be helpful toward them or that they are unsure of whether their peer is being helpful or not. The button to the left side of the steering wheel was identified as an analogue for children to indicate that the peer is being hostile toward them.

In order to assist participants in using the buttons, a graphical representation of the meaning of the button appeared on the screen when it was pressed. These graphics included a smiley face on the right side of the screen next to the opponent's car and an angry face on the left side of the opponent's car. Previous research employing a nonverbal response dial with children has used "smiley face" graphics to imply positive and negative responses, and found that this method may assist children in making a nonverbal, evaluative response (Parisi, 2004). To indicate that the right smiley face button was pushed, the face turned from grey to yellow in color, which is the standard color for smiley faces. When the left angry face was pressed, the graphic turned from grey to red, indicating anger. A brief instruction period was also completed to ensure that children memorized the difference between the left and right buttons. If participants were exhibiting difficulty in this area, they were corrected until they demonstrated an understanding of the function of the buttons.

All instances of pushing the button were sent electronically to the computer and recorded via interface with the racing program (Loewald, 2009). Specifically, the time at which each button was pressed was recorded in a text file which was later entered into a database. The software program also indicated whether the left or right button was pressed. As mentioned 
before, responses were elicited using a stimulus graphic at eight crash prompts and at eight noncrash prompts. Instances in which the left-hand button was pushed within 10 seconds of a prompt were counted as hostile attributions, while right-hand button pushes were counted as non-hostile. If both buttons were pushed in the span of 10 seconds following a prompt, the button pushed first was counted. The goal of the nonverbal procedure was to measure the number of prompts to which a hostile attribution was made, even if only briefly. As the computer game was not designed to measure the length of time that a button was pressed, this data was not collected. The computer data resulted in a count of hostile attributions made across the race, with the maximum being 16 times. Most participants did not respond at each of the 16 prompts, so the rate of hostile attribution responses was calculated in addition to the number. Percentages of hostile attribution responses were created by dividing the number of hostile attribution responses by the total number of responses a participant made. This provided a score that is comparable across participants. Percentage scores were also calculated for crash prompts and non-crash prompts by dividing the number of hostile attributions made at crashes or in the absence of crashes by the total number of crash or non-crash responses.

Verbal measure of real-time hostile attribution bias. Verbal protocol analysis (Ericsson \& Simon, 1980) was used to measure the interpretations that participants have about the fictional peers with whom they are interacting in the computer task. Verbal protocol analysis involves asking the participants to verbally express what is occurring while they are processing a situation. As with the nonverbal measure of hostile attributions, the response cue was displayed on the screen indicating that the participant should be responding. Instead of pressing a button when the cue appeared, children were instructed to talk about whether their opponent was trying to help them win the game or keep them from winning the game while the cue appeared on the 
screen. Following the participant's response, his or her statements were coded based on their content.

For the current study, children's protocols were analyzed at each of the 16 prompts during the racing period. Responses during the verbal race were recorded either on an audio cassette or digitally on the computer. They were later transcribed and the content of children's responses was matched with the time, in seconds, that the response cue appeared on the screen. In this way, each verbal response was matched with the game event of one of the eight crash prompts or one of the eight non-crash cues. If a response occurred within 10 seconds of the response cue, it was counted as a response to that cue. Some responses fell outside of those parameters and were not coded at all.

In reviewing the verbal protocol, a coding procedure used by Milch-Reich and colleagues (1999) was modified to target coding of attributions. Milch-Reich and colleagues' (1999) coding procedure counted the number of concepts that participants identified in their protocol as an analogue for measuring encoding. Concepts were defined as objects or people that children discussed in their protocols. Milch-Reich and colleagues (1999) looked for verbal cues indicating that a participant was making an inference, or an attribution. They noted that their coding scheme was designed to be particularly sensitive given the possibility that their young participants may have less advanced verbal skills. They used cues in responses such as "the word because or inferring a feeling state" to indicate that an inference was being made (Milch-Reich et al., 1999). These ideas were also used in the coding of verbal protocols in the current study.

The coding scheme for this study also relied on the theories of hostile attribution bias (Crick \& Dodge, 1994; Dodge, 2006) as well as on the content of participants' verbal protocols (see Table 1). In particular, it was proposed that the current study use codes for hostile and non- 
hostile intent. Attribution of hostile intent codes were divided into those that included statements of 1) hostile intent with opponent trying to win, 2) hostile intent with opponent trying to crash, 3) observation of a crash, and 4) opposite of benign intent (e.g. "He is NOT helping me.”). Several neutral codes were created, which included 5) observation of opponent's poor performance and 6) neutral comments. Attributions of non-hostile, or benign, intent fell into 4 categories: 7) opposite of hostile intent (e.g. "He is NOT hitting me.”), 8) unintentional or accidental, 9) participant's acceptance of blame, or 10) benign or helpful intent. In addition to these codes, which categorized responses that directly indicated attribution of intent, other codes reflected related cognitions.

A number of verbal protocols included self-reflections and meta-cognitions which sometimes contained expressions of emotions, especially anger. Seven additional codes were created to capture these cognitions. Some responses included statements in which participants expressed their own hostile intent or aggressive action either 11) to retaliate against a perceived threat or 12) for no stated reason. When participants expressed their anger, they usually commented that it was 13) a result of their opponent's behavior or 14) caused by the game itself. Additionally, participants made several other types of statements including 15) providing an instruction or a command, 16) making an affective exclamation, or 17) reporting their own benign intent. In order to determine reliability of coding, responses from $20 \%$ of the sample $(n=$ 14) were re-coded by another doctoral student familiar with the project. Interrater reliability (kappa $=.78)$ was found to be adequate.

The codes which most clearly indicated hostile attribution of intent were those that stated 1) the opponent was trying to win, 4) the opponent was NOT being helpful, and 11) the participant was aggressing because he or she perceived that the opponent was being hostile. 
Table 1

Real-time Verbal Response Coding Scheme

\begin{tabular}{|c|c|}
\hline Intent Codes & Exemplars \\
\hline $\begin{array}{l}\text { 1.Hostile Intent w/goal of winning } \\
\text { race }\end{array}$ & - "He is trying to make me lose" \\
\hline 2.Hostile intent w/goal of crashing & $\begin{array}{l}\text { - "He's trying to run me over" } \\
\text { - "He just wrecked me." }\end{array}$ \\
\hline 3.Observation of crash & $\begin{array}{l}\text { - "Oh, she got on top of me." } \\
\text { - "She is crashing into me." }\end{array}$ \\
\hline 4.NOT benign intent & - "She is NOT helping me." \\
\hline 5.Poor Performance & $\begin{array}{l}\text { - "He is doing bad." } \\
\text { - "He can't drive." }\end{array}$ \\
\hline 6.Neutral Comment & $\begin{array}{l}\text { - "We are racing fine." } \\
\text { - "It won't stay off the wall." }\end{array}$ \\
\hline 7.NOT hostile intent & - "He's NOT hitting me." \\
\hline 8.Unintentional/Accidental & - "It was just an accident" \\
\hline 9.Acceptance of Blame & - "Oh my bad." \\
\hline 10. Benign/Helpful Intent & $\begin{array}{l}\text { - "She's being nice." } \\
\text { - "He's helping me." } \\
\text { - "Now we are working as a team." } \\
\text { - "I think he's helping me by trying to stay out of } \\
\quad \text { my way and go for the lead." }\end{array}$ \\
\hline Affective (Self-Reflection) Codes & Exemplars \\
\hline 11. Aggression for retaliation & $\begin{array}{l}\text { - "I'm being an angry player, because she hit me." } \\
\text { - "He almost cut me off. Stupid self. I'm about to } \\
\text { bump him." }\end{array}$ \\
\hline 12. Aggression (no reason stated) & $\begin{array}{l}\text { - "I'm trying to beat her now." } \\
\text { - "Yeah! Got him!" }\end{array}$ \\
\hline $\begin{array}{l}\text { 13. Anger or frustration caused by } \\
\text { opponent }\end{array}$ & - "He's making me mad." \\
\hline $\begin{array}{l}\text { 14. Anger or frustration caused by } \\
\text { game or another explanation }\end{array}$ & $\begin{array}{l}\text { - "I just don't know how to drive. It's frustrating." } \\
\text { - "I hate when that happens." } \\
\text { - "This game is hard man." }\end{array}$ \\
\hline 15. Command/Instructions & $\begin{array}{l}\text { - "Stop trying to run into me." } \\
\text { - "That car need to move!" }\end{array}$ \\
\hline 16. Affective exclamation & $\begin{array}{l}\text { - "Uh, dang it!" } \\
\text { - "Come on!" } \\
\text { - "Oh no!" } \\
\end{array}$ \\
\hline 17. Benign action & - "I'm being a helpful player." \\
\hline
\end{tabular}


Additionally, code 13, in which the participant expresses his or her anger due to action of the opponent, was also included as theoretically related to hostile attribution because the participant expresses his or her perception that the opponent is a threat. Indeed, responses coded 13 and coded 11 were significantly correlated $(r=.41, p<.001)$. When adding code 13 to codes 1,4 , and 11, an affective element is introduced resulting in a score that reflects the participants' hostile/angry attributions. Codes 2 and 3 both commented on the crashes occurring and theoretically may have seemed to indicate hostile intent, though analyses indicated that neither related to aggression.

Following analysis of the protocol for each provocation, the total number of responses coded $1,4,11$, or 13 were calculated, representing the score for the number of hostile/angry attributions each participant made. Because not every participant responded to every prompt, the count of hostile/angry responses are less comparable between participants than the percentage of hostile/angry attributions. The count of hostile/angry responses was divided by the total number of responses for each participant, which resulted in the percentage of each participant's responses that included hostile/angry attributions. The percentage of hostile/angry attributions was used as the measure of verbal hostile/angry attribution in analyses.

Several analyses were also conducted using the percentage of verbal hostile/angry attributions made in response to crash versus non-crash prompts. In these cases, the score was calculated by dividing the number of responses to crash prompts coded $1,4,11$, or 13 by the total number of responses to crash prompts. Similarly, the percentage for non-crash prompts was calculated by dividing the number of hostile/angry attributions at non-crash prompts by the total number of responses to non-crash prompts. 
Vignette measure of hostile attribution bias. Many commonly used methods of testing hostile attribution biases in children use vignettes adapted from those created by Dodge (1980; Dodge, Pettit, McClaskey, Brown, \& Gottman, 1986; Erdley \& Asher, 1998; Lochman \& Dodge, 1994). The Home Interview with Child was used as the traditional measure of hostile attribution because of its widely-used social information-processing vignettes (see Appendix; Conduct Problems Prevention Research Group, 1991; Dodge, Bates, \& Pettit, 1990). This measure presents eight vignettes involving ambiguous peer interactions, which are read aloud to participants. The measure asks children to imagine that they are participating in the interaction, which involves some negative action by peers. It is ambiguous in the scenarios whether the negative action was intentionally harmful or accidental. Participants are asked to explain why the provocation occurred in an open-ended response format. Of the eight vignettes in the measure, four vignettes include Ambiguous Minor Harm, such as a peer spilling something on the participant. The other four vignettes involve Unsuccessful Peer Entry. In these vignettes, the participant imagines that he or she attempts to enter a group and is not responded to.

Previous studies have coded responses to the open-ended question into one of three categories: nonhostile, hostile or don't know. However, the coding scheme was expanded for this study to include more possible categories of response. The coding scheme was based on concepts of coding verbal protocols outlined by Malle, Knobe, \& Nelson (2007). Malle's (2007) method of coding narratives for attributions notes distinct differences in language used to suggest an intentional attribution versus an unintentional attribution. Verbs suggesting intent attributions are termed "mental state markers" (e.g. want, like, need, hope, trying, think). An example of an attribution of hostile intent using a mental state marker is "He bumped into me because he wanted to beat me in the race." Not all attributions of hostile intent have mental state markers 
(e.g. "He bumped into me because he is losing"), however, mental state markers were used as a guide to help identify attributions of intent. A coding scheme was developed and revised using exemplars, or clear examples, of codes derived from responses given by participants in the pilot study (see Table 2). Responses were coded as indicating that the person in the vignette acted: 1) with hostile intent with instrumental goals, 2) with hostile intent as a reaction, 3) unintentionally/accidentally, 4) with benign intent, or 5) with ambiguous intent. Additional codes were created for scenarios in which responses suggested: 6) the intent was unknown, 7) both hostile (1 or 2$)$ and non-hostile $(3,4$, or 5$)$ intents were mentioned, but hostile intents were primary, and 8 ) both hostile (1 or 2$)$ and non-hostile $(3,4$, or 5$)$ intents were mentioned, but nonhostile intents were primary. A hostile or non-hostile intent was determined to be "primary" if it was emphasized, or, in cases where both were equally emphasized, it was given first. Each 8response transcript was coded by the author according to this scheme. In order to determine reliability of coding, responses from $20 \%$ of the sample $(n=15)$ were re-coded by another doctoral student familiar with the project. Inter-rater reliability $($ kappa $=.78)$ was found to be adequate.

Codes were collapsed such that each code was determined to indicate hostile or nonhostile attributions. If a response was coded with a 1,2 , or 7 , it was counted as a hostile attribution, while other codes were counted as non-hostile attributions. The total number of hostile attributions across the eight vignettes was calculated, as were the number of hostile attributions made on the two, 4-item scales - Ambiguous Minor Harm and Unsuccessful Peer Entry. Scores on the two scales were significantly correlated $(r=.34, p<.01)$. The internal consistency of the total scale was moderate $(\alpha=.65)$. Because of the conceptual and statistical 
Table 2

Hostile Attribution Bias (HAB) Vignette Measure Coding Scheme

Code

Exemplars (from pilot data)

1. Intentionally hostile (proactive):

a. to bully, to be mean

b. for personal gain

c. To be rude or unfriendly

- Because she is trying to act cool in front of her friends. She probably likes you but she is trying to act cool.

- Because usually when you are a new kid, they don't like you, so they be mean.

- She was probably a bully and she was probably being mean.

2. Intentionally hostile (reactive):

a. hostile intent as retaliation

b. an act of jealousy

- He was afraid I might be a bully; he didn't want to be bullied.

- She was jealous because you had brand new sneakers and she didn't.

3. Nonintentional - accident:

a. Could be due to unavoidable external forces

- I don't think he did it on purpose. It probably was an accident because he was in a hurry trying to get where he was going.

- There was too many of them

- Because they were too busy, they might not have noticed me.

4. Intentionally non-hostile helping

a. intent was benign or to

- To get my attention be helpful

5. Intentional - neutral or ambiguous

a. Not overtly hostile or retaliatory
- They don't know me.

- Because usually the first day of school, they sit around and be quiet and are usually shy.

- Because he's new at that club and they don't want to say nothing. They just want to see how you do and get to know you.
6. Unsure of intent

7. Both hostile and non-hostile responses - hostile primary
- I don't know.

- Either he was doing something else or he just didn't want to play because he was not that nice. I would guess he was trying to probably be mean because I didn't see him doing nothing. If some people say no to me and I don't see them doing nothing, I think they are trying to be mean

- He was either being mean or he was trying to go somewhere and accidentally bumped me. But if he tried to push me out of the way to get to the drink machine, it would probably be on purpose. (which one?) I wouldn't guess he probably didn't try to do it because if someone does that to me, they would probably say I'm sorry or they would try to make fun of me and stuff. (which one?) I think it's an accident. 
relationship between the two scales, the total score combining both scales was used in analyses as the vignette-based measure of hostile attribution.

Measure of aggression. This study measures how hostile attribution measurements relate to child aggression. While the child participant completed the racing game and hostile attribution measure, his or her parent completed the Parent-rating scale for Reactive and Proactive Aggression (PRPA; see Appendix; Kempes, Matthys, Maassen, van Goozen, \& van Engeland, 2006). This 22 -item measure asked parents to rate their child on a scale ranging from 1 ("Never True") to 5 ("Almost Always True") in items of proactive and reactive aggression. It was adapted from the teacher version of the measure (Dodge \& Coie, 1987). Half of the 22 items measure proactive aggression and half measure reactive aggression resulting in two subscales of 11 items each.

A recent confirmatory factor analysis of the PRPA suggested that a shortened version, including only 11 items, is a better measure of aggressive behavior than the 22 -item version (Kempes et al., 2006). In the current study, the 22-item version and the 11-item subset were compared. Reliabilities of both scales were good; internal consistencies of the 22-item and 11item scales were .93 and .86 respectively. Kempes and colleagues (2006) asserted that the 11item measure more clearly differentiated between reactive and proactive aggression than the 22item version, because it excludes some items found to be ambiguous. Given this, the 11-item version was chosen for use in the current analyses.

The positive correlation between reactive and proactive aggression has been wellestablished (Card \& Little, 2006; Kempes et al., 2006; Polman et al., 2007), though the two often differentially relate to variables of children's well-being (Card \& Little, 2006; Dodge \& Coie, 
1987; Dodge, Lochman, Harnish, Bates, \& Pettit, 1997; Kempes et al., 2006; Poulin \& Boivin, 2000). Indeed, when examining the factor structure of the PRPA, Kempes and colleagues (2006) determined that a two-factor solution (reactive and proactive scales) was preferable to a single factor, despite the strong correlation between the two factors. Some literature has dealt with the distinction between reactive and proactive aggression by partialling out the portion of reactive aggression explained by proactive aggression, and the portion of proactive aggression that covaries with reactive aggression (Poulin \& Boivin, 2000). In the current study, reactive and proactive aggression using the 11-item PRPA were highly positively correlated $(r=.65, p<$ .001). To better measure the unique relations between hostile attributions and reactive or proactive aggression, analyses will use partial correlations and multiple regression to control for reactive aggression when predicting proactive aggression, and to control for proactive aggression when predicting reactive aggression.

Video game experience measure. In order to determine if a child's personal experience with video games influenced his or her performance on the task, a self-report measure of children's video game play was administered. This technique is commonly used in research about aggression in video games to control for individual differences in video game experience (Anderson \& Dill, 2000; Gentile et al., 2009). Children were asked to list their three favorite video games and were then asked how often they played each game. Previous research has used a 7-point scale with anchors of rarely at point 1, occasionally at point 4, and often at point 7 in samples of college students (Anderson \& Dill, 2000). This study used an open-ended question of how often participants played each identified game. If children were unsure of how to answer, they were provided with anchors of once a day, a few times per week, once a week, a few times per month, or once a month to assist them in being more specific. Responses were then coded on 
a scale of 0 to 9 with higher numbers representing more frequent play. Video games were coded 1 if they included a racing component and 0 if they did not. The frequency score was multiplied by the number of games (out of the 3 games) named that included racing component. The result was a score representing how often each participant played racing games, ranging from 0 to 27 . This score was used in exploratory analyses to test the effect of frequent racing game play on performance in this study. 


\section{CHAPTER 3}

\section{RESULTS}

\section{Descriptive and Demographic Analyses}

Means and standard deviations were calculated for each of the dependent variables and the independent variables (see Table 3). On the 11-item PRPA, possible scores ranged from 0 to $44(M=11.78, S D=6.63)$. Subscale scores of reactive and proactive aggression were significantly correlated $(r=.65, p<.001)$. Of the two subscales on the PRPA, parents reported significantly more reactive aggression $(M=8.32, S D=4.43)$ than proactive aggression $(M=$ $3.46, S D=2.83 ; t(73)=12.41, p<.001)$.

On the vignette measure of hostile attribution, children's responses ranged from 0 to $8(M$ $=3.97, S D=2.08$ ). The two scales comprising the vignette measure of hostile attributions were significantly correlated $(r=.35, p<.01)$. Participants made significantly more hostile attributions on the Ambiguous Minor Harm scale $(M=2.15, S D=1.27)$ than on the Unsuccessful Peer Entry scale $(M=1.81, S D=1.25 ; t(74)=2.00, p=.05)$.

Real-time hostile attribution measure. Using the nonverbal response procedure of the real-time measure of hostile attribution, participants made an average of $11.24(S D=3.79)$ responses out of 16 total prompts, meaning that they responded an average of $68.75 \%$ of the 
Table 3

Means and Standard Deviations for Variables

\begin{tabular}{|c|c|c|c|c|c|}
\hline & & $N$ & Range & $M$ & $S D$ \\
\hline \multirow{3}{*}{$\begin{array}{c}\text { PRPA } \\
(11 \text {-items })^{\mathrm{a}}\end{array}$} & Reactive & \multirow{3}{*}{74} & $0-24$ & 8.32 & 4.43 \\
\hline & Proactive & & $0-20$ & 3.46 & 2.83 \\
\hline & Total & & $0-44$ & 11.78 & 6.63 \\
\hline \multirow{3}{*}{$\begin{array}{c}\text { HAB } \\
\text { Vignette } \\
\text { Measure }\end{array}$} & Ambiguous Minor Harm & \multirow{3}{*}{75} & $0-4$ & 2.15 & 1.27 \\
\hline & Unsuccessful Peer Entry & & $0-4$ & 1.81 & 1.25 \\
\hline & Total & & $0-8$ & 3.96 & 2.06 \\
\hline \multirow{5}{*}{$\begin{array}{l}\text { Real-time } \\
\text { Nonverbal } \\
\text { Responses }\end{array}$} & Total Number of Responses & 75 & $0-16$ & 11.24 & 3.79 \\
\hline & \# of Hostile Attributions & 72 & $0-16$ & 5.76 & 3.38 \\
\hline & $\%$ of Hostile Attributions & 72 & $0-100$ & 51.02 & 28.71 \\
\hline & $\%$ of Host. Attr. At Crashes & 72 & $0-100$ & 61.28 & 31.06 \\
\hline & $\%$ of Host. Attr. At Non-Crashes & 72 & $0-100$ & 40.51 & 33.19 \\
\hline \multirow{5}{*}{$\begin{array}{l}\text { Real-time } \\
\text { Verbal } \\
\text { Responses }\end{array}$} & Total Number of Responses & 75 & $0-16$ & 9.43 & 4.46 \\
\hline & \# of Hostile/Angry Attributions & 72 & $0-16$ & 1.56 & 2.03 \\
\hline & $\%$ of Hostile/Angry Attributions & 72 & $0-100$ & 16.23 & 22.32 \\
\hline & $\%$ of Host. Attr. At Crashes & 72 & $0-100$ & 14.87 & 23.53 \\
\hline & $\%$ of Host. Attr. At Non-Crashes & 72 & $0-100$ & 15.45 & 24.83 \\
\hline
\end{tabular}

${ }^{\mathrm{a}}$ Responses were on a 5-point scale, scored from 0 to 4.

time. Of those responses, an average of $51.02 \%(S D=28.71 \%)$ reflected a hostile attribution $(M$ $=5.76, S D=3.38)$. The remaining responses indicated non-hostile attribution of intent $(M=$ $5.94, S D=3.96)$. The count of nonverbal hostile attributions was significantly negatively correlated with the count of nonverbal non-hostile attributions $(r=-.66, p<.001)$. Participants averaged $3.33(S D=1.92)$ hostile attributions on the eight prompts occurring after crashes, which represents an average $61.28 \%(S D=31.06)$ of total responses to crash prompts. The mean of hostile attributions made after the eight prompts occurring at non-crashes was $2.43(S D=$ $2.01)$, which is an average of $40.51 \%(S D=33.19)$ of total responses to non-crash prompts. While the percentage of hostile attributions made in response to crash prompts was significantly 
higher than that made in response to non-crash prompts $(t(71)=5.87, p<.001)$, the two percentages were also positively correlated $(r=.57, p<.001)$.

On the verbal response protocols of the real-time measure of hostile attribution, participants provided a response to an average of $58.92 \%$ of the 16 prompts $(M=9.43, S D=$ 4.46). This reflects participants' tendency to respond to prompts in the nonverbal race more frequently than to those in the verbal race $(t(74)=2.76, p=.007)$. Participants made an average of $1.56(S D=2.03)$ hostile/angry attributions across 16 prompts, which was an average of $16.23 \%(S D=22.32)$ of responses. It should be noted that this rate was significantly lower than the rate of hostile responses given using the nonverbal response procedure $(t(68)=14.49, p<$ .001). When presented with eight prompts occurring after crashes in the game, hostile/angry attributions were made an average of $.75(S D=1.21)$ times, or $14.87 \%(S D=23.53)$ of total responses to crash prompts. Hostile/angry attributions at eight non-crash prompts $(M=.81, S D$ $=1.24)$ were made at a similar frequency $(M=15.45 \%, S D=24.83)$. As in the nonverbal response procedure, participants who made hostile/angry attributions in response to prompts after crashes were more likely to also make those attributions in response to non-crash prompts $(r=$ $.37, p=.001)$

Demographic differences. Means of different demographic groups were compared using $t$-tests to determine if their scores differed significantly on measures of hostile attribution or aggression. No significant differences were found between boys and girls in the sample with respect to their mean levels of aggression, including reactive and proactive aggression, or hostile attributions including scores on the vignette measure, verbal protocol, and nonverbal protocol. 
Similarly, no significant differences were found between mean scores of variables in African American and Caucasian ethnic groups.

The location where participants completed the study was examined as a factor in their responses. Participants who completed the study in the lab $(M=10.12 \%, S D=14.30 \%)$ setting showed a lower percentage of verbal responses reflecting hostile/angry attributions ( $M=19.90 \%$, $S D=25.42 \% ; t(70)=-2.09, p=0.04)$. To explore this difference, correlations between the percentage of verbal hostile/angry attributions and outcome variables were compared for participants completing the study in the lab versus in their home. Similar patterns of effects were found between participants who completed the study in the lab versus in their home, suggesting that differences in the rate of hostile/angry attributions did not influence their relation to outcomes.

Participants' age was explored in relation to their hostile attribution responses, as well as parent-rated aggression. Age was negatively correlated with the number of verbal hostile/angry attributions made by participants $(r=-.25, p=.04)$, meaning that younger children made more hostile/angry attributions. Age was also correlated with the total number of verbal responses given $(r=-.25, p=.03)$, which indicates that younger children also gave more responses overall. When the percentage of verbal responses that were hostile/angry attributions were calculated, they did not significantly relate to age $(r=-.08, p=.49)$, suggesting that the relation between age and the number of verbal hostile/angry attributions can be accounted for by differences in base rates of responses across ages. 


\section{Hypotheses Tests}

Analyses were completed to test the hypotheses of the project. Specifically, relations between the two new real-time measurements of hostile attributions and aggression were tested. The combination of the two real-time measures was also tested using regression to determine if the two together was a better predictor than either measure alone. The relations between realtime hostile attributions and proactive and reactive aggression were also tested. Relations between the vignette measure of hostile attribution and aggression were also explored. The predictive ability of the new, real-time measures was compared with the traditional, vignette measure of hostile attribution using regression.

Hypothesis 1. Correlations revealed that the percentage of nonverbal responses reflecting hostile attributions was not correlated with aggression $(r=.04, p=.73)$. When examining the percentage of hostile attributions in nonverbal responses to crash and non-crash prompts, neither score was significantly correlated with aggression $(r=-.002, p=.99$, and $r=$ $.07, p=.57$, respectively).

Using the verbal response procedure, the percentage of responses reflecting hostile/angry attributions trended toward significant correlation with parent-rated aggression $(r=.21, p=.08)$. The percentage of verbal hostile/angry attributions in response to prompts after crashes was positively correlated with aggression $(r=.28, p=.02)$. In response to non-crash prompts, the percentage of verbal hostile/angry attributions was not correlated with aggression $(r=.12, p=$ .31). 
Hypothesis 2. As different effects were predicted between the real-time measure of attributions and two forms of aggression - reactive and proactive - these were tested using partial correlations to control for the other form of aggression. Partial correlation of the percentage of nonverbal hostile attribution with reactive aggression was significant $(r=.28, p=$ .02 ) after controlling for proactive aggression. Participants with higher reactive aggression scores made more hostile attributions using nonverbal responses, when the variability accounted for by proactive aggression was removed. The opposite effect was observed for proactive aggression; after controlling for reactive aggression, the percentage of nonverbal hostile attributions was significantly negatively correlated with proactive aggression $(r=-.29, p=.02)$.

When using the verbal response procedure, partial correlations controlling for proactive aggression also suggested a positive relation between reactive aggression and the percentage of hostile/angry attributions made $(r=.24, p=.05)$. Proactive aggression was not significantly related to verbal hostile/angry attributions after controlling for reactive aggression using partial correlations $(r=-.08, p=.51){ }^{1}$

Hypothesis 3. The combination of verbal and nonverbal scores was measured by centering and averaging the nonverbal hostile attribution percentage and the verbal hostile/angry attribution percentage. This new combination score represented a real-time hostile attribution score. When partialling out proactive aggression, this score was significantly positively related to reactive aggression $(r=.33, p=.007)$. An opposite effect was found when measuring the

\footnotetext{
${ }^{1}$ Exploratory analyses were completed using zero-order correlations to determine the relation between verbal and nonverbal hostile attributions and reactive and proactive aggression, without controlling for either (see Table 4). Results suggested that nonverbal hostile attributions were not significantly related to reactive or proactive aggression. Verbal hostile/angry attributions were not significantly related to proactive aggression, but did significantly relate to reactive aggression $(r=.25, p=.03)$.
} 
Table 4

Zero-Order Correlations between Independent and Dependent Variables

\begin{tabular}{|c|c|c|c|c|c|c|c|c|c|c|c|c|c|c|c|c|c|c|}
\hline \multirow[b]{2}{*}{ Scale } & \multirow[b]{2}{*}{ Subscale } & & \multicolumn{3}{|c|}{$\begin{array}{c}\text { PRPA } \\
\text { (11-items) }\end{array}$} & \multicolumn{3}{|c|}{$\begin{array}{c}\text { HAB Vignette } \\
\text { Measure }\end{array}$} & \multicolumn{5}{|c|}{ Nonverbal Hostile Attributions } & \multicolumn{5}{|c|}{ Verbal Hostile/Angry Attributions } \\
\hline & & & 1 & 2 & 3 & 1 & 2 & 3 & 1 & 2 & 3 & 4 & 5 & 1 & 2 & 3 & 4 & 5 \\
\hline \multirow{3}{*}{$\begin{array}{l}\text { PRPA } \\
(11 \text {-items })^{\mathrm{a}}\end{array}$} & Reactive & 1 & - & $.65^{*}$ & $.95 *$ & .03 & -.08 & -.03 & .06 & .15 & .15 & .08 & .18 & -.07 & $.22^{\dagger}$ & $.25^{*}$ & $.27 *$ & .18 \\
\hline & Proactive & 2 & & - & $.86^{*}$ & .08 & -.02 & .03 & .05 & -.08 & -.13 & -.12 & -.12 & -.06 & .12 & .10 & $.23^{\dagger}$ & .00 \\
\hline & Total & 3 & & & - & .05 & -.06 & -.01 & .06 & .07 & .04 & .00 & .07 & -.07 & $.20^{\dagger}$ & $.21^{\dagger}$ & $.28 *$ & .12 \\
\hline \multirow{3}{*}{$\begin{array}{l}\text { HAB Vignette } \\
\text { Measure }\end{array}$} & Minor Harm & 1 & & & & - & $.34 *$ & $.82 *$ & .02 & -.14 & -.18 & -.11 & -.20 & -.06 & .07 & -.05 & .02 & .02 \\
\hline & Peer Entry & 2 & & & & & - & $.82 *$ & .02 & .11 & .001 & .05 & -.01 & .13 & .15 & -.03 & .13 & -.09 \\
\hline & Total & 3 & & & & & & - & .03 & -.02 & -.11 & -.03 & -.13 & .04 & .13 & -.05 & .09 & -.04 \\
\hline \multirow{5}{*}{$\begin{array}{l}\text { Real-time } \\
\text { Nonverbal } \\
\text { Hostile } \\
\text { Attributions }\end{array}$} & \# of Responses & 1 & & & & & & & - & $.25^{*}$ & $-.24 *$ & -.10 & $-.21^{\dagger}$ & .05 & .07 & -.02 & .12 & -.05 \\
\hline & \# of Host. Attr. & 2 & & & & & & & & - & $.85^{*}$ & $.76^{*}$ & $.78 *$ & -.07 & .17 & .14 & $.23^{\dagger}$ & .07 \\
\hline & $\%$ of Host. Attr. & 3 & & & & & & & & & - & $.85^{*}$ & $.89 *$ & -.18 & .15 & $.23^{\dagger}$ & .17 & .17 \\
\hline & $\%$ at Crashes & 4 & & & & & & & & & & - & $.57 *$ & -.13 & .12 & .12 & .14 & .07 \\
\hline & $\%$ at Non-crashes & 5 & & & & & & & & & & & - & -.17 & .11 & $.23^{\dagger}$ & .13 & .17 \\
\hline \multirow{5}{*}{$\begin{array}{l}\text { Real-time } \\
\text { Verbal } \\
\text { Hostile/Angry } \\
\text { Attributions }\end{array}$} & \# of Responses & 1 & & & & & & & & & & & & - & $.33^{*}$ & -.04 & .13 & -.05 \\
\hline & \# of Host. Attr. & 2 & & & & & & & & & & & & & - & $.79 *$ & $.77 *$ & $.68^{*}$ \\
\hline & $\%$ of Host. Attr. & 3 & & & & & & & & & & & & & & - & $.71 *$ & $.88 *$ \\
\hline & $\%$ at Crashes & 4 & & & & & & & & & & & & & & & - & $.37 *$ \\
\hline & $\%$ at Non-crashes & 5 & & & & & & & & & & & & & & & & - \\
\hline
\end{tabular}

$* p<.05$

$p<.01$ 
relation between the combination score and proactive aggression, after partialling out reactive $\operatorname{aggression}(r=-.24, p=.05)$.

The unique effect of the nonverbal and verbal attributions together on proactive and reactive aggression was assessed. Multiple regression revealed that nonverbal hostile attributions predicted reactive aggression $(\beta=.23, t(68)=2.59, p=.01)$ above and beyond that which was predicted by proactive aggression $(\beta=.67, t(68)=7.46, p<.001)$. Sequential regression (see Table 5) was performed to determine how the addition of verbal response scores changed the ability to predict reactive aggression. The addition of verbal response scores to the model did not result in a significant change in $\mathrm{R}$-squared $\left(\Delta \mathrm{R}^{2}=.019, \Delta F(1,64)=2.20, p=.14\right)$. This suggests that verbal response scores do not significantly improve ability of nonverbal responses to predict reactive aggression, after controlling for proactive aggression.

The effect of adding nonverbal response scores to the model in which verbal scores predicted reactive aggression, after controlling for proactive aggression, was examined. Multiple regression revealed that the percentage of verbal hostile/angry attributions predicted reactive aggression $(\beta=.19, t(68)=2.12, p=.04)$ above and beyond that which was predicted by proactive aggression $(\beta=.62, t(68)=6.77, p<.001)$. Sequential regression was performed to determine how the addition of the nonverbal response scores changed the ability to predict reactive aggression. The addition of the percentage of nonverbal hostile attributions to the model resulted in a change in $\mathrm{R}$-squared that trended toward significance $\left(\Delta \mathrm{R}^{2}=.032, \Delta F(1,64)=\right.$ $3.73, p=.06)$. This suggests that the percentage of nonverbal hostile attributions may have an additive effect on the ability of verbal hostile/angry attributions to predict reactive aggression, after controlling for proactive aggression. 
Table 5

Hypothesis 3: Sequential Set Regression

\begin{tabular}{|c|c|c|c|c|c|c|}
\hline DV & Step & IV & $\beta$ & $t$ & $p$ & $\mathrm{R}^{2}$ \\
\hline \multirow{5}{*}{$\begin{array}{c}\text { Reactive } \\
\text { Aggression }\end{array}$} & \multirow{2}{*}{1} & Proactive Aggression & .66 & 7.01 & $.00 *$ & \multirow{2}{*}{.439} \\
\hline & & Nonverbal Hostile Attribution & .22 & 2.34 & $.02 *$ & \\
\hline & \multirow{3}{*}{2} & Proactive Aggression & .64 & 6.83 & $.00 *$ & \multirow{3}{*}{$.458^{1}$} \\
\hline & & Nonverbal Hostile Attribution & .19 & 1.93 & .06 & \\
\hline & & Verbal Hostile/Angry Attr. & .14 & 1.48 & .14 & \\
\hline \multirow{5}{*}{$\begin{array}{c}\text { Reactive } \\
\text { Aggression }\end{array}$} & \multirow{2}{*}{1} & Proactive Aggression & .61 & 6.47 & $.00 *$ & \multirow{2}{*}{.426} \\
\hline & & Verbal Hostile/Angry Attr. & .19 & 1.98 & $.05 *$ & \\
\hline & \multirow{3}{*}{2} & Proactive Aggression & .64 & 6.83 & $.00 *$ & \multirow{3}{*}{$.458^{2}$} \\
\hline & & Verbal Hostile/Angry Attr. & .14 & 1.48 & .14 & \\
\hline & & Nonverbal Hostile Attribution & .19 & 1.93 & .06 & \\
\hline
\end{tabular}

${ }^{*} p<.05$

${ }^{1} \Delta \mathrm{R}^{2}=.019, \Delta F(1,64)=2.20, p=.14$

${ }^{2} \Delta \mathrm{R}^{2}=.032, \Delta F(1,64)=3.73, p=.06$

Hypothesis 4. In order to test whether verbal and nonverbal real-time measures of hostile attribution are better able to predict reactive aggression than are traditional, vignette measures of hostile attribution, a standard multiple regression was performed with reactive aggression as the dependent variable, and proactive aggression as a covariate. The independent variables included in the model were the combination score of the percentages of verbal and nonverbal real-time hostile attributions and the hostile attribution score from the vignette measure. Results indicated that this collection of variables was able to explain a significant amount of the variance in reactive aggression $\left(\mathrm{R}^{2}=.46, F(3,64)=18.10, p<.001\right)$. The realtime measures of hostile attribution significantly predicted reactive aggression $(\beta=.25, t(64)=$ 2.73, $p<.01)$, whereas the vignette measure $\operatorname{did} \operatorname{not}(\beta=-.04, t(64)=-.44, p=.66)$. 


\section{Power analyses}

Power analyses were performed prior to the start of the study, which determined that a sample size of 75 would allow adequate power, given the varied analyses needed across the four hypotheses. With this sample size, the power to detect an effect of the same size found in previous studies ( $r=.55$; Orobio de Castro et al., 2002) of the relation between hostile attribution and aggression using staged situations (Hypothesis 1) would be $\alpha>.99$. The effect predicted was much smaller than the effect found, which was non-significant for the relation between nonverbal hostile attributions and aggression. For the relation between verbal hostile/angry attributions and aggression, which trended to significance, the post hoc power analysis indicated $\alpha=.57$, suggesting an increased sample size could have resulted in a significant effect.

For Hypotheses $2 \& 3$, the relations between real-time hostile attribution scores and reactive aggression has not been studied previously, though literature has shown the effect size of the relation between hostile attributions and both aggression and reactive aggression are comparable (Orobio de Castro et al., 2002). In light of this, it was expected that real-time attribution scores will also relate to reactive aggression on a magnitude similar to that of their relation with aggression, making the effect size and power similar to $r=.55$ (Orobio de Castro et al., 2002) and $\alpha>.99$, respectively. Post hoc power analyses reveal that, using partial correlation rather than zero-order correlation, the test of the relation between nonverbal hostile attributions and reactive aggression had a power of $\alpha=.70$ and between nonverbal hostile attributions and proactive aggression had a power of $\alpha=.74$. For partial correlations of verbal hostile/angry attributions and reactive aggression, post hoc analyses suggested the power to detect an effect was $\alpha=.56$. For the partial correlation between verbal hostile/angry attributions and proactive aggression, the power was $\alpha=.11$. In the test of the third hypothesis, the partial correlation of 
the combination of verbal and nonverbal hostile attributions and reactive aggression of $\mathrm{r}=.33$ corresponded with a power of $\alpha=.56$. The power of the partial correlation between the combination score and proactive aggression was $\alpha=.85$.

Finally, for the fourth hypothesis, no previous literature has tested the ability of the new measures to better predict aggression beyond that predicted by traditional measures. Given a medium effect size and a sample size of 75 , the power using a test of R-squared in a set regression was predicted to be $\alpha=.85$. Post hoc analyses for this test indicate a power of $\alpha=.99$.

\section{Exploratory Analyses}

Order effects. When comparing participants who completed the nonverbal procedure first with those who completed the verbal procedure first, some order effects were discovered. The percentage of nonverbal hostile attributions was significantly greater for participants completing the nonverbal race first, in comparison with those who completed it after the verbal race $(t(70)=2.04, p=.05)$. This difference seemed to also contribute to different relations between nonverbal hostile attributions and reactive aggression depending on the order in which participants completed the races. For children who completed the nonverbal protocol during their first race, the percent of hostile attributions they made correlated with reactive aggression $(r=$ $.48, p<.01)$, after controlling for proactive aggression. If children completed the nonverbal protocol second, the percent of hostile attributions was not significantly correlated with reactive aggression $(r=.13, p=.50)$. In spite of this, these two correlations were not significantly different $(Z=1.58, p=.11)$. Nonverbal hostile attributions remained a significant predictor of reactive aggression, even after controlling for order effects and proactive aggression in a multiple regression $(\beta=.24, t=2.62, p=.01)$. In addition, when tests of the hypotheses described above 
were revised to control for order effects, no changes in the significance of the findings were discovered. Therefore, while order effects may have resulted in different partial correlations for the relation between reactive aggression and nonverbal hostile attributions, they do not appear to be strong enough to change the nature of the findings in the whole sample.

Vignette measure. Effects between the vignette measure used in this study and reactive and proactive aggression have been found in previous studies. Due to the lack of effect found in this study, the characteristics of the vignette measure were explored. Each of the eight items was examined and none had a significant correlation with parent-rated aggression. In order to further assess the vignette measure, teacher ratings of aggression using the Teacher Observation of Child Adaptation - Revised (TOCA-R) were examined. These ratings were collected as part of the larger study of the Coping Power program from which this sample was recruited. Ratings were available for 63 of the 75 participants and had been collected prior to participants' completion of the current study. Responses to one vignette were correlated with TOCA-R scores. In response to vignette F (see Appendix), an Ambiguous Minor Harm vignette, participants who made hostile attributions had higher teacher-rated aggression as measured by the TOCA-R Authority Acceptance scale $(r=.30, p=.02)$.

Race segment. The timing of prompts during the 5 minute real-time measure of hostile attribution was randomly selected. The randomization resulted in parts of the race having a number of prompts in quick succession and other parts of the race having no prompts. To explore response patterns throughout the race, the 5 minute race was divided into five segments based on sequential groups of prompts (see Table 6). Using the nonverbal response procedure, participants' hostile attributions made during the Late segment of the race were significantly 
correlated with overall aggression $(r=.25, p=.04)$ as well as reactive aggression $(r=.33, p<$ $.01)$. Nonverbal responses on other segments of the race were not significantly related to aggression. Verbal hostile/angry attributions in each race segment were not significantly related to aggression or reactive aggression.

Table 6

\begin{tabular}{lll} 
Race Segments & & \\
\hline Segment & Time & Prompt \\
\hline First & $0: 09$ & Crash 1 \\
Early & $0: 45$ & Non-crash 1 \\
& $1: 17$ & Non-crash 2 \\
& $1: 23$ & Non-crash 3 \\
& $1: 36$ & Crash 2 \\
& $1: 54$ & Crash 3 \\
Middle & $2: 48$ & Non-crash 4 \\
& $3: 00$ & Crash 4 \\
& $3: 08$ & Crash 5 \\
Late & $3: 56$ & Crash 6 \\
& $4: 07$ & Non-crash 5 \\
& $4: 25$ & Non-crash 6 \\
& $4: 35$ & Non-crash 7 \\
& $4: 49$ & Non-crash 8 \\
Last & $4: 53$ & Crash 7 \\
& $4: 59$ & Crash 8 \\
\hline
\end{tabular}

Video game play. Scores were collected from each participant to rate how often they played racing games in their daily life. The effect of racing game play was explored in relation to the predictor variables and the outcomes in the current study. Racing game play was not significantly correlated with either the verbal or nonverbal real-time attribution measures, nor was it significantly correlated with scores on the vignette measure of hostile attributions. Scores 
of reactive and proactive aggression were not significantly correlated with participants' racing game play.

Manipulation effects. Among participants, two acknowledged during the racing game that they believed they were playing against a computer rather than a peer. Two more participants mentioned following the debriefing process that they thought maybe they had been playing against a computer. The means of the four participants were not significantly different from the means of the entire sample on the verbal real-time attribution score, the nonverbal realtime attribution score, the vignette measure attribution score, the reactive aggression score, or the proactive aggression score. To test whether the inclusion of these participants into analyses affected the results, hypothesis tests described above were completed again after removing the scores of these four participants. Exclusion of these four participants did not change the significance of the results above, suggesting that those who guessed they were playing against a computer rather than a peer did not respond differently from those who did. 


\section{CHAPTER 4}

\section{DISCUSSION}

This results of this study support a new way of conceptualizing hostile attributions that occur in real time. Real-time hostile attributions were measured using a new verbal and nonverbal procedure expected to significantly relate to aggression (Hypothesis 1). Results indicate that only verbal hostile/angry attributions following crashes were significantly related to aggression. The lack of significant relation between verbal and nonverbal real-time hostile attribution and aggression is due to their differential relationships with reactive aggression and proactive aggression. As predicted, partial correlations indicated a significant positive relationship between the nonverbal hostile attribution score and reactive aggression, after controlling for proactive aggression. Proactive aggression, after controlling for reactive aggression, was also related to nonverbal hostile attributions, but in the negative direction. For the verbal procedure, results supported Hypothesis 2. Verbal hostile/angry attributions were significantly positively related to reactive aggression, after controlling for proactive aggression, but were not significantly related to proactive aggression.

In support of Hypothesis 3, the combination of the verbal and nonverbal measures into one real-time hostile attribution score was significantly positively related to reactive aggression, after controlling for proactive aggression. The significant negative relation between proactive aggression and hostile attributions that was found in the nonverbal procedure was also found 
when the verbal and nonverbal scores were combined; which is further evidence that real-time hostile attributions differentially predict reactive and proactive aggression.

Finally, the new real-time measures were compared with a traditional, vignette- based measure of hostile attribution. As predicted, the new measures were able to significantly predict reactive aggression, whereas the vignette measure was not significantly related to reactive aggression.

\section{Real-time Hostile Attributions}

This study describes and tests the concept of real-time hostile attributions, which builds upon the appraisal processes explained in models of social cognition, including the social information-processing model (Crick \& Dodge, 1994). Real-time hostile attributions are the interpretations made in the moment, as a social interaction occurs. Unlike other characterizations of hostile attributions, they do not appear to require conscious, deliberative thought; yet, they are not wholly automatic cognitions. This can be seen in the responses using the real-time verbal procedure, which sometimes included statements reflecting rational reasoning about the opponents' attribution. At other times, participants responded with an impulsive, affective exclamation. Regardless of how much reasoning was put into the attribution, it was measured in the context of a game that required a portion of the participant's attention.

This mimics the parallel cognitive and behavioral processes of real social interactions in which children are expected to take in and interpret social information simultaneously while engaging in social behavior. These demands are unique to real-time interactions and cannot be measured using vignettes. Young (2010) studied encoding, or paying attention to and taking in certain cues, that occurs immediately before the interpretation, or attribution, is made. She noted 
that encoding is preconscious and developed a real-time measure of encoding using reaction times to assess aggressive youth's attention bias toward angry versus happy facial expressions. These results, coupled with the results of the current study, suggest that real-time appraisal processes likely work in concert with one another with reactively aggressive children showing an attentional bias to aggressive cues and an attributional bias toward hostile interpretations. While this sequence has been described as a deliberative process in the social information-processing model, these new ways of thinking about real-time appraisal processes suggest that they occur automatically and seamlessly.

Given the divided attention required during social interactions, real-time hostile attributions likely rely on heuristics and schemas, as well as short term memories, more than traditional conceptualizations of hostile attributions. This was evidenced when participants indicated hostile attributions in the racing game even when crashes were not occurring, suggesting that these rapid cognitions were based on internal rather than external cues. Real-time hostile attribution measurement targets these internal processes in a way that vignettes cannot. Even in response to distinct provocation, such as in a racecar crash, the real-time hostile attributions were often based on internal cues, especially anger and physiological arousal. As a result, real-time hostile attributions reflect individual differences more than hostile attributions as traditionally measured. Real-time hostile attribution measurement appears to provide a new level of understanding of what actually occurs while a child engages in social interactions.

Exploratory analyses indicated that the relation between nonverbal hostile attributions and reactive aggression was especially strong when participants completed the nonverbal race before the verbal race. For the group that completed the verbal race first, the relation between 
nonverbal hostile attributions and reactive aggression had diminished and was no longer significant during the nonverbal race. These findings suggest that the order of the races was important, however, when additional analyses were run controlling for order effects, no changes to the results were observed. The nonverbal response procedure may be more vulnerable to practice effects than the verbal response procedure because of its reliance on heuristic, nonverbal information. It is possible that once participants completed the verbal race, they had developed a heuristic of the role of the opposing car as hostile. This may have then been accessed during the nonverbal response race that followed. Interestingly, this effect was not seen in the verbal response procedure suggesting that the attributions made during the first race using the nonverbal procedure were not conscious or accessible during the second race using the verbal response procedure.

\section{Validity of Real-Time Hostile Attribution Measurement}

The new real-time measures are better able to examine the appraisal process because they show an improvement in ecological validity over the traditional, vignette-based measures. First, participants were personally involved in the interaction in which they made hostile attributions. Vignettes only allow participants to be observers or imaginarily involved in the interaction used to test hostile attributions. The game used in the real-time measure closely mimics video games that children play in their everyday lives, often in head-to-head competition with peers as in this study. The response procedure mirrors the in-the-moment social information-processing that is required of youth as they interact with their peers on an everyday basis. In contrast, vignette measures allow for prolonged interpretation and consideration of all options, which was especially apparent in this study when vignette responses included both hostile and non-hostile 
attributions. The ecological validity of this task is consistent with the ideas presented by Orobio de Castro and colleagues (2002) suggesting that measurement using staged situations would produce the strongest effect between hostile attribution and aggression. This validity likely accounts for the finding that the real-time measures are more strongly related to reactive aggression than the vignette-based measure.

The real-time hostile attributions measured in this study are good predictors of both proactive and reactive aggression. The differential results for the nonverbal procedure in predicting reactive versus proactive aggression suggest strong discriminant validity of the construct of real-time hostile attributions and the procedure used to measure them. This also explains why the nonverbal hostile attribution score was not significantly related to the overall aggression score, which was a combination of reactive and proactive aggression items. It appears that the nonverbal procedure captures a type of hostile attribution that is particularly predictive of reactive aggression and its contrast to proactive aggression. The verbal hostile/angry attributions also showed discriminant validity in that they are significantly related to reactive aggression, but showed no relation to proactive aggression.

Both the verbal and nonverbal response procedures appear to be measuring hostile attributions that relate to reactive aggression, but they differ in their relation to proactive aggression. Nonverbal responses were negatively related to proactive aggression suggesting that the hostile attributions targeted by this procedure are qualitatively different from verbal hostile/angry attributions. The nonverbal hostile attributions reflected responses made to a request to choose between two polarized options - hostile or non-hostile attributions. This forced choice may capture the immediate, reactive nature of reactive aggression, as opposed to the more intentional and planned-out characteristics associated with proactive aggression. This may 
explain why nonverbal hostile attributions were negatively related with proactive aggression. The verbal procedure seems to capture the reactive response, especially as it involves anger arousal. Verbal responses also allow for some explanation of reasoning, which could diminish any negative effect found between hostile attributions and proactive aggression. While the two procedures responded to identical provocations, it is clear that they were measuring distinct aspects of real-time hostile attributions.

\section{The Role of Emotion in Hostile Attributions}

In the verbal response procedure, the emotion of anger was an important part of hostile/angry attributions, which were related to reactive aggression. Anger has been included in previous models of social information-processing. Specifically, the anger cues exhibited by peers are thought to be used to develop hostile attributions (Lemerise \& Arsenio, 2000). This study controls for the emotional cues of the peer because the "peer" was automated and standard across participants. The individual differences in hostile/angry attributions in this study come from participants' internal anger cues. This suggests that physiological arousal cues were a key part of the formation of verbal hostile/angry attributions. Previous research has indicated that moderately aggressive boys who perceived a threat showed an increase in hostile attributions along with an increase in heart rate (Williams, Lochman, Phillips, \& Barry, 2003). The results of this study provide additional evidence that increased emotional arousal is associated with increased hostile attributions, and their relation is likely bidirectional. The verbal hostile/angry attribution can then be viewed as a unique measure of the co-occurrence of the hostile attribution and associated emotional arousal. 
The concept of the verbal hostile/angry attribution closely matches the theoretical processes underlying reactive aggression, and not proactive aggression. Reactive aggression is believed to be driven by anger associated with a perceived threat, and has been shown to relate to anger and physiological arousal (Hubbard et al., 2002). The findings in this study support the relation of anger and physiological arousal to both reactive aggression and hostile attributions simultaneously. This was further supported by findings that the relation between reactive aggression and nonverbal hostile attributions were significant in later portions of the race, but not in earlier portions of the race. Emotional and physiological arousal could increase throughout the course of the race and result in more hostile attributions toward the end. Future research could use empirical methods of measuring physiological arousal in order to further test these suspected effects on real-time hostile attributions. For example, tests of skin galvanization or heart rate could provide information about sympathetic nervous system activity as it occurs in real-time with hostile attributions.

\section{Limitations}

As with all studies based on correlational analyses, the direction of the relation between the independent variable and the dependent variable could be interpreted differently than hypothesized. The hypotheses in this study posit that hostile attributions predict aggressive behavior, which is based on the theory underlying the social information-processing model. As indicated in the model, it is likely that the two variables are bidirectionally related. Aggression may also be predictive of hostile attributions. Children who display aggression may have a tendency to make a hostile attribution in order to justify their aggressive behavior. This selfserving attribution could reflect a "top-down" approach to social information-processing, 
whereby aggressive behavior influences the subsequent appraisal process. The direction of the relation between aggressive behavior and hostile attribution is difficult to determine, especially given the real-time, rapid processing that occurs when making an attribution during a social interaction. Future research could explore the attribution-aggression link in several ways. Realtime hostile attributions could be measured using the procedure in this study at several different points over a period of time. Parent-rated aggressive behavior could also be measured at these times to help determine if hostile attributions are associated with a change in aggressive behavior over time, or if aggressive behavior is associated with a change in hostile attributions over time. Alternatively, real-time hostile attributions could be compared with real-time aggressive behavior.

In the current study, aggressive behavior is measured using retrospective parent report of children's behavior, while hostile attributions are measured in real time. In order to explore the direction of the effect between aggression and hostile attributions, aggressive behavior could be measured within the same game used to elicit hostile attributions. Using the current procedure, aggression, in the form of deliberate crashes in the racing game, is difficult to measure, because it is unclear if participants intentionally or unintentionally caused crashes. In addition, some children might have tried to cause a crash, or otherwise be aggressive, but were not successful. Future research could more clearly measure real-time aggression by providing a mechanism for children to be deliberately aggressive during the game. Other literature has used games in which buttons can be pushed to provoke or annoy an opponent (e.g. Phillips \& Lochann, 2003). Analyses comparing this type of aggression during the game with parent-rated aggression could provide additional dimensions to our understanding of the link between aggression and real-time hostile attributions. 
The analyses in this study use one particular method of predicting reactive and proactive aggression by controlling for the other. The result is a test of the ability of the real-time hostile attributions to predict the portion of reactive aggression not accounted for by proactive aggression, and the portion of proactive aggression not accounted for by reactive aggression. While some have argued that proactive and reactive aggression should be examined together because they often co-occur in a child, a strong body of literature suggests that many risk factors, including hostile attributions, relate differentially to proactive and reactive aggression (Card \& Little, 2006; Dodge \& Coie, 1987; Dodge et al., 1997; Kempes et al., 2006; Poulin \& Boivin, 2000). The results of this study provide further support for the idea that it is helpful to partial proactive aggression out of reactive aggression to better understand the unique predictive ability of these two types of aggression. Future research should explore the specific aspects of reactive and proactive aggression that relate to one another, as well as the variables that might explain their divergence.

The vignette-based measure used in this study is commonly used in literature to measure hostile attributions. The responses to the measure were coded using a rubric developed for the current study, whereas previous research has categorized responses into 3 groups (hostile, nonhostile, and don’t know) or used multiple choice responses rather than open-ended responses. The response differences could explain why the measure was not found to be related to aggression in this sample, though some literature using the same measure has also failed to find a significant relation with aggression (Hubbard et al., 2002). Future research should continue to use the measure with a variety of measures of aggression to determine if differences might exist in its' relation with parent-rated (as in the current study), teacher-rated, or observed aggressive behavior. 
An alternative explanation for the lack of significant findings using the vignette measure could be due to the intervention sample. The participants had all been part of a larger study which had engaged them in a preventive intervention that included skills training on perspectivetaking and examining all possible attributions in a situation (Lochman \& Wells, 2002). This may have resulted in participants using their skills when presented with the vignettes in this study. The sample in this study may have been more deliberative; in other words, they could have been more likely to examine all possible attributions and less likely to display a hostile attribution bias on the vignette measure. Additionally, the participants in this sample had been exposed to other vignette measures as part of the evaluation for the preventive intervention study. They could have been accustomed to thinking through a vignette more than participants in a sample who had never completed a vignette-based measure. Given these unique characteristics of this sample, future studies could find stronger effects between vignette measures and aggressive behavior.

The real-time measures, as well as the traditional, vignette-based measures, of hostile attribution use unknown or ambiguous peers, which does not account for dyadic factors between a child and a known peer. Many acts of aggression in children are in response to interactions with peers they know and with whom they have a history of interaction. Patterns of interactions and schemas of their relationships are likely to guide peers' attributions about one another (Hubbard et al., 2001). These relationship-specific attributions are difficult to measure in the research setting. Instead, this and other studies rely on generalized measures of hostile attribution to make predictions about aggressive behavior. 


\section{Implications}

This study provides new evidence of the importance and uniqueness of real-time measures of social information-processing. While a number of researchers have called for more studies with real-time measurement (e.g. Crick \& Dodge, 1994; Milch-Reich et al., 1999; Fontaine, 2008), few have developed or tested such measures. Real-time measurement of social information-processing should be expanded to include measures of social goals, response generation and evaluation, and aggressive behavior as they occur. The current procedure could be replicated to test other social cognitions. For example, children could be asked to explain their goals aloud to create a verbal protocol of social goals as they occur in real-time. While it was important to use an ambiguous provocation to test hostile attribution in the real-time measure, future studies could use more overtly aggressive provocations. Just as blasts of noise and tilting a game have been used as analogues of aggressive behavior in previous studies (Phillips \& Lochman, 2003), intentional crashes or road blocks created by the opponent in the racing game could create threatening situations to test children's responses in research settings.

The real-time measures in this study could allow researchers and clinicians to better identify children who are at risk for reactive aggression. As we are better able to predict aggressive reactions in children, we can more effectively target children who most need preventive intervention. The nonverbal procedure alone could help to determine which children are most likely respond to a perceived threat with reactive aggression. The measure may be particularly appealing to researchers and clinicians because of its precision, as well as its ease in administration. The procedure takes only 5 minutes to administer and children appear to enjoy the game. It also produces results free from potential biases found in parent-report and teacher- 
report measures. Once children are identified as at-risk, they could be administered preventive interventions designed to address their particular risk.

The current findings suggest that real-time hostile attributions are important, not only in predicting aggression, but also in preventing aggression. Preventive interventions designed to reduce aggression, especially reactive aggression, could be more effective by targeting real-time hostile attributions. The findings emphasize the importance of helping children change social cognitions as they happen in the moment. Because real-time hostile attributions happen quickly and with less deliberative thought, interventions could focus on helping children slow this process. Interventions might teach children to recognize the types of ambiguous social situations that often result in hostile attributions. If children are able to increase their metacognition about the appraisal process, they might be more likely to consider other attributions of intent besides hostile attributions.

The real-time attribution procedures presented in this study could be used to assist in targeting hostile attributions in preventive intervention. As outlined above, the procedure is particularly appealing because it actively engages kids in an enjoyable task that is also brief and easy to administer. In intervention, the procedure could be used to practice attending to instances when one is making a hostile attribution. Children could also play the game with the goal of thinking through their attributions deliberately while still engaging in the task. This could help kids practice dividing their attention and thinking about their social cognitions while interacting socially. Some children might also benefit from interventions that examine their responses to a baseline measurement of real-time hostile attributions. Children's responses could be used as a starting point in discussing hostile attributions and the importance of stopping to 
think through the possible intentions of a peer. In particular, verbal responses could be examined to help children work through their reasoning and to highlight the role that anger and physiological arousal might play in hostile attributions. Some or all of these techniques could be added to existing attribution retraining interventions or could be used as a standalone intervention.

In conclusion, the results of the current study provide evidence, not only for new measures of hostile attribution, but also for new conceptualizations of hostile attributions as they occur in real time. Real-time attributions appear to be particularly important in predicting reactive and proactive aggression. The verbal and nonverbal response procedures each provide a unique piece of the real-time attribution picture, with verbal responses emphasizing the role of anger and physiological arousal. In the future, the real-time hostile attribution procedure could be used to identify children who would benefit most from targeted interventions. These preventive interventions could build on existing attribution retraining procedures to help children attend to and change their hostile attributions in real time. 


\section{REFERENCES}

Abramson, L. Y., Metalsky, G. I., \& Alloy, L. B. (1989). Hopelessness depression: A theorybased subtype of depression. Psychological Review, 96, 358-372. doi: 10.1037/0033295X.96.2.358

Ajzen, I. (1991). The theory of planned behavior. Organizational Behavior and Human Decision Processes, 50, 179-211. doi: 10.1016/0749-5978(91)90020-T

Ajzen, I., \& Fishbein, M. (1980). Understanding attitudes and predicting social behavior. Englewood Cliffs, NJ: Prentice-Hall.

Alwitt, L.F. (2002). Suspense and advertising responses. Journal of Consumer Psychology, 12, 35-49. doi: 10.1207/S15327663JCP1201_04

Anderson. C. A., \& Dill, K. E. (2000). Video games and aggressive thoughts, feelings, and behavior in the laboratory and in life. Journal of Personality and Social Psychology, 78, 772-790. doi: 10.1037/0022-3514.78.4.772

Armitage, C. J., \& Conner, M. (2001). Efficacy of the theory of planned behavior: A metaanalytic review. British Journal of Social Psychology, 40, 471-499. doi:

$10.1348 / 014466601164939$

Arsenio, W.F., Adams, E., Gold, J. (2009). Social information processing, moral reasoning, and emotion attributions: Relations with adolescents reactive and proactive aggression. Child Development, 80, 1739-1755. doi: 10.1111/j.1467-8624.2009.01365.x

Atkins, M. S., Stoff, D. M., Osborne, M. L., \& Brown, K. (1993). Distinguishing instrumental and hostile aggression: Does it make a difference? Journal of Abnormal Child Psychology, 21, 355-365. doi: 10.1007/BF01261598

Bailey, C. A., \& Ostrov, J. M. (2008). Differentiating forms and functions of aggression in emerging adults: Associations with hostile attribution biases and normative beliefs. Journal of Youth and Adolescence, 37, 713-722. doi: 10.1007/s10964-007-9211-5

Bandura, A. (1973). Aggression: A social learning analysis. Oxford, England: Prentice-Hall.

Berkowitz, L. (1989). Frustration-aggression hypothesis: Examination and reformulation. Psychological Bulletin, 106, 59-73. doi: 10.1037/0033-2909.106.1.59 
Berndt, T.J. (1979). Developmental changes in conformity to peers and parents. Developmental Psychology, 15, 608-616. doi: 10.1037/0012-1649.15.6.608

Blyth, D.A., Simmons, R.G., \& Bush, D. (1978) The transition into early adolescence: A longitudinal comparison of youth in two educational contexts. Sociology of Education, 51, 149-162. doi: $10.2307 / 2112661$

Bosacki, S. L., Marini, Z. A., \& Dane, A. V. (2006). Voices from the classroom: Pictorial and narrative representations of children's bullying experiences. Journal of Moral Education, 35, 231-245. doi: 10.1080/03057240600681769

Card, N.A. \& Little, T.D. (2006). Proactive and reactive aggression in childhood and adolescence: A meta-analysis of differential relations with psychosocial adjustment. International Journal of Behavioral Development, 30, 466-480. doi: 10.1177/0165025406071904.

Conduct Problems Prevention Research Group (CPPRG). (1991). Home Interview with Child. Unpublished instrument. Retrieved from http://www.fasttrackproject.org/

Crick, N. R. (1995). Relational aggression: The role of intent attributions, bfeelings of distress, and provocation type. Development and Psychopathology, 7, 313-322. doi: $10.1017 / \mathrm{S} 0954579400006520$

Crick, N. R., \& Dodge, K. A. (1994). A review and reformulation of social informationprocessing mechanisms in children's social adjustment. Psychological Bulletin, 115, 74101. doi: $10.1037 / 0033-2909.115 .1 .74$

Crick, N. R., \& Dodge, K. A. (1996). Social information-processing mechanisms on reactive and proactive aggression. Child Development, 67, 993-1002. doi: 10.2307/1131875

Crick, N. R., \& Ladd, G. W. (1990). Children's perceptions of the outcomes of social strategies: Do the ends justify being mean? Developmental Psychology, 26, 612-620. doi: 10.1037/0012-1649.26.4.612

Dodge, K. A. (1980). Social cognition and children's aggressive behavior. Child Development, 51, 162-170. doi: 10.2307/1129603

Dodge, K. A. (2006). Translational science in action: Hostile attributional style and the development of aggressive behavior problems. Development and Psychopathology, 18, 791-814. doi: 10.1017/S0954579406060391

Dodge, K. A. (2008) On the meaning of meaning when being mean: Commentary on Berkowitz's "On the consideration of automatic as well as controlled psychological processes in aggression." Aggressive Behavior, 34, 133-135. doi:10.1002/ab.20242. 
Dodge, K. A., Bates, J. E., \& Pettit, G. S. (1990). Mechanisms in the cycle of violence. Science, 250, 1678-1683. doi: 10.1126/science.2270481

Dodge, K. A., \& Coie, J. D. (1987). Social-information-processing factors in reactive and proactive aggression in children's peer groups. Journal of Personality and Social Psychology, 53, Special issue: Integrating personality and social psychology. 1146-1158. doi: 10.1037/0022-3514.53.6.1146

Dodge, K. A., \& Frame, C. L. (1982). Social cognitive biases and deficits in aggressive boys. Child Development, 53, 620-635. doi: 10.2307/1129373

Dodge, K. A., Lochman, J. E., Harnish, J. D., Bates, J. E., \& Pettit, G. S. (1997). Reactive and proactive aggression in school children and psychiatrically impaired chronically assaultive youth. Journal of Abnormal Psychology, 106, 37-51. doi: 10.1037/0021843X.106.1.37

Dodge, K. A., \& Pettit, G. S. (2003). A biopsychosocial model of the development of chronic conduct problems in adolescents. Developmental Psychology, 39, 349-371. doi: 10.1037/0012-1649.39.2.349

Dodge, K. A., Pettit, G. S., McClaskey, C. L., \& Brown, M. M. (1986). Social competence in children. Monographs of the Society for Research in Child Development, 51, 1-85. doi: 10.2307/1165906

Dodge, K.A. \& Somberg, D.R. (1987). Hostile attributional biases among aggressive boys are exacerbated under conditions of threats to the self. Child Development, 58, 213-224. doi: $10.2307 / 1130303$

Dorsch, A., \& Keane, S. P. (1994) Contextual factors in children's social information processing. Developmental Psychology, 30, 611-616. doi: 10.1037/0012-1649.30.5.611

Eccles, J. S., Midgley, C., Wigfield, A., Buchanan, C. M., Reuman, D., Flanagan, C., \& MacIver, D. (1993). Development during adolescence: The impact of stage-environment fit on young adolescents' experiences in schools and in families. American Psychologist, 48, 90-101. doi: 10.1037/ 66X.48.2.90

Erdley, C. A., \& Asher, S. R. (1996). Children's social goals and self-efficacy perceptions as influences on their responses to ambiguous provocation. Child Development, 67, 13291344. doi: $10.2307 / 1131703$

Erdley, C. A., \& Asher, S. R. (1998). Linkages between children's beliefs about the legitimacy of aggression and their behavior. Social Development, 7, 321-339. doi: 10.1111/14679507.00070 
Ericsson, K.A., \& Simon, H.A. (1980). Verbal reports as data. Psychological Review, 87, 215251. doi: 10.1037/0033-295X.87.3.215

Fite, P. J., Colder, C. R., Lochman, J. E., \& Wells, K. C. (2006). The mutual influence of parenting and boys' externalizing behavior problems. Applied Developmental Psychology, 27, 151-164. doi: 10.1016/j.appdev.2005.12.011

Fontaine, R. G. (2008). On-line social decision making and antisocial behavior: Some essential but neglected issues. Clinical Psychology Review, 28, 17-35. doi:

10.1016/j.cpr.2007.09.004

Fontaine, R. G., \& Dodge, K. A. (2006). Real-time decision making and aggressive behavior in youth: A heuristic model of response evaluation and decision (RED). Aggressive Behavior, 32, 604-624. doi: 10.1002/ab.20150

Fredrickson, W. E. (1997). Elementary, middle, and high school student perceptions of tension in music. Journal of Research in Music Education, 45, 626-635. doi: 10.2307/3345427

Gentile, D. A., Anderson, C. A., Yukawa, S., Ihori, N., Saleem, M., Ming, L. K., ...Sakamoto, A. (2009). The effects of prosocial video games on prosocial behaviors: International evidence from correlational, longitudinal, and experimental studies. Personality and Social Psychology Bulletin, 35, 752-763. doi: 10.1177/0146167209333045

Gilbert, D. T., \& Malone, P. S. (1995). The correspondence bias. Psychological Bulletin, 117, 21-38. doi: 10.1037/0033-2909.117.1.21

Graziano, W. G., Moore, J. S., \& Collins, J. E. (1988). Social cognition as segmentation of the stream of behavior. Developmental Psychology, 24, 568-573. doi: 10.1037/00121649.24.4.568

Horsley, T.A., Orobio de Castro, B., \& Van der Schoot, M. (2010). In the eye of the beholder: Eye-tracking assessment of social information processing in aggressive behavior. Journal of Abnormal Child Psychology, 38, 587-599. doi: 10.1007/s10802-009-9361-x

Hubbard, J. A. (2005). Eliciting and measuring children's anger in the context of their peer interactions: Ethical consideration practical guidelines. Ethics \& Behavior, 15, 247-258. doi: 10.1207/s15327019eb1503_5

Hubbard, J.A., Dodge, K.A., Cillessen, A.H. N., Coie, J.D., \& Schwartz, D. (2001). The dyadic nature of social information processing in boys' reactive and proactive aggression. Journal of Personality and Social Psychology, 80, 268-280. doi: 10.1037/00223514.80.2.268 
Hubbard, J.A., Smithmyer, C.M., Ramsden, S.R., Parker, E.H., Flanagan, K.D., Dearing, K.F., ...Simons, R.F. (2002). Observational, physiological, and self-report measures of children's anger: Relations to reactive versus proactive aggression. Child Development, 73, 1101-1118. doi: 10.1111/1467-8624.00460

Huesmann, L. R., \& Guerra, N. G. (1997). Children's normative beliefs about aggression and aggressive behavior. Journal of Personality and Social Psychology, 72, 408-419. doi: 10.1037/0022-3514.72.2.408

Kempes, M., Matthys, W., Maassen, G., van Goozen, S., \& van Engeland, H. (2006). A parent questionnaire for distinguishing between reactive and proactive aggression in children. European Child and Adolescent Psychiatry, 15, 38-45. doi: 10.1007/s00787-006-0502-2

Kupersmidt, J. B., \& Patterson, C. J. (1991). Childhood peer rejection, aggression, withdrawal, and perceived competence as predictors of self-reported behavior problems in preadolescence. Journal of Abnormal Child Psychology, 19, 427-449. doi: 10.1007/BF00919087

Lansford, J. E., Malone, P. S., Dodge, K. A., Crozier, J. C., Pettit, G. S., \& Bates, J. E. (2006). A 12-year prospective study of patterns of social information processing problems and externalizing behaviors. Journal of Abnormal Child Psychology, 34, 715-724. doi: 10.1007/s10802-006-9057-4

Leff, S. S., Crick, N. R., \& Angelucci, J., Haye, K., Jawad, A. F., Grossman, M., \& Power, T. J. (2006). Social cognition in context: Validating a cartoon-based attributional measure for urban girls. Child Development, 77, 1351-1358. doi: 10.1111/j.1467-8624.2006.00939.x

Lemerise, E.A. \& Arsenio, W.F. (2000). An integrated model of emotion processes and cognition in social information processing. Child Development, 71, doi: 10.1111/14678624.00124

Lochman, J. E., \& Dodge, K. A. (1994). Social-cognitive processes of severely violent, moderately aggressive, and nonaggressive boys. Journal of Consulting and Clinical Psychology, 62, 366-374. doi: 10.1037/0022-006X.62.2.366

Lochman, J. E., \& Dodge, K. A. (1998). Distorted perceptions in dyadic interactions of aggressive and nonaggressive boys: Effects of prior expectations, context, and boys' age. Development and Psychopathology, 10, 495-512. doi: 10.1017/S0954579498001710

Lochman, J.E., \& Lenhart, L. (1995). Cognitive behavioral therapy of aggressive children: Effects of schemas. In H.P.J.G. van Bilsen, P.C. Kendall, \& J.H. Slavenburg (Eds.), Behavioral approaches for children and adolescents: Challenges for the next century (pp. 145-166). New York: Plenum Press. 
Loewald, T. (2009). Racer [Computer software]. Retrieved from http://www.loewald.com/racer/

Lorber, M. (2007). Validity of video-mediated recall procedures for mothers' emotion and child ratings. Journal of Family Psychology, 21, 520-528. doi: 10.1037/0893-3200.21.3.520

Mackinnon-Lewis, C., Lamb, M. E., Hattie, J., Baradaran, L. P. (2001). A longitudinal examination of the associations between mothers' and sons' attributions and their aggression. Development and Psychopathology, 13, 69-81. doi: $10.1017 / \mathrm{S} 0954579401001055$

Malle, B. F. (2007). F.Ex: Coding scheme for people's folk explanations of behavior. University of Oregon. Retrieved February 3, 2009, from http://darkwing.uoregon.edu/ interact/fex.html (Original work published 1998)

Malle, B. F., Knobe, J. M., \& Nelson, S. E. (2007). Actor-observer asymmetries in explanations of behavior: New answers to an old question. Journal of Personality and Social Psychology, 93, 491-514. doi: 10.1037/0022-3514.93.4.491

Mezulis, A. H., Abramson, L. Y., Hyde, J. S., \& Hankin, B. L. (2004). Is there a universal positivity bias in attributions? A meta-analytic review of individual, developmental, and cultural differences in the self-serving attributional bias. Psychological Bulletin, 130, 711-747. doi: 10.1037/0033-2909.130.5.711

Milch-Reich, S., Campbell, S. B., Pelham, W. E. Jr., Connelly, L. M. \& Geva, D. (1999). Developmental and individual differences in children's real-time representations of dynamic social events. Child Development, 70, 413-431. doi: 10.1111/1467-8624.00030

Miller, D. T., \& Norman, S. A. (1975). Actor-observer differences in perceptions of effective control. Journal of Personality and Social Psychology, 31, 503-515. doi: $10.1037 / \mathrm{h} 0076485$

Nas, C. N., Orobio de Castro, B., \& Koops, W. (2005). Social information processing in delinquent adolescents. Psychology, Crime \& Law, 11, 363-375. doi: $10.1080 / 10683160500255307$

Ohan, J. L., \& Johnston, C. (2007). What is the social impact of ADHD in girls? A multi-method assessment. Journal of Abnormal Child Psychology, 35, 239-250. doi: 10.1007/s10802006-9076-1

Orobio de Castro, B., Merk, W., Koops, W., Veerman, J.W., \& Bosch, J.D. (2005). Emotions in social information processing and their relations with reactive and proactive aggression in referred aggressive boys. Journal of Clinical Child and Adolescent Psychology, 34, 105116. doi: $10.1207 / \mathrm{s} 15374424$ jccp3401_10 
Orobio de Castro, B., Slot, N.W., Bosch, J.D., Koops, W., \& Veerman, J.W. (2003). Negative feelings exacerbate hostile attributions of intent in highly aggressive boys. Journal of Clinical Child and Adolescent Psychology, 32, 56-65. doi: 10.1207/S15374424JCCP3201_06

Orobio de Castro, B., Veerman, J. W., Koops, W., Bosch, J. D., \& Monshouwer, H. J. (2002). Hostile attribution of intent and aggressive behavior: A meta-analysis. Child Development, 73, 916-934. doi: 10.1111/1467-8624.00447

Ouelette, J. A., \& Wood, W. (1998). Habit and intention in everyday life: The multiple processes by which past behavior predicts future behavior. Psychological Bulletin, 124, 54-74. doi: 10.1037/0033-2909.124.1.54

Parisi, J. (2004). Fourth- and fifth-grade students' affective response and ability to discriminate between melody and improvisation after receiving instruction in singing and/or playing a piece in the blues style. International Journal of Music Education, 22, 77-86. doi: $10.1177 / 0255761404042376$

Perry, D. G., Perry, L. C. \& Rasmussen, P. (1986). Cognitive social learning mediators of aggression. Child Development, 57, 700-711. doi: 10.2307/1130347

Phillips, N. C., \& Lochman, J. E. (2003). Experimentally manipulated change in children's proactive and reactive aggressive behavior. Aggressive Behavior, 29, 215-227. doi: 10.1002/ab.10028

Polman, H., Orobio de Castro, B., Koops, W., van Boxtel, H.W., \& Merk, W.W. (2007). A metaanalysis of the distinction between reactive and proactive aggression in children and adolescents. Journal of Abnormal Child Psychology, 35, 522-535. doi: 10.1007/s10801007-909-4

Poulin, F. \& Boivin, M. (2000). Reactive and proactive aggression: Evidence of a two-factor model. Psychological Assessment, 12, 115-122. doi: 10.1037//1040-3590.12.2.115

Robins, R. W., Spranca, M. D. \& Mendelsohn, G. A. (1996). The actor-observer effect revisited: Effects of individual differences and repeated social interactions on actor and observer attributions. Journal of Personality and Social Psychology, 71, 375-389. doi: 10.1037/0022-3514.71.2.375

Runions, K.C. \& Keating, D.P. (2010). Anger and inhibitory control as moderators of children's hostile attributions and aggression. Journal of Applied Developmental Psychology, 31, 370-378. doi: 10.1016/j.appdev.2010.05.006

Steinberg, M. S., \& Dodge, K. A. (1983). Attributional bias in aggressive adolescent boys and girls. Journal of Social \& Clinical Psychology, 1, 312-321. 
Strack, F., \& Deutsch, R. (2004). Reflective and impulsive determinants of social behavior. Personality and Social Psychology Review, 8, 220-247. doi:

10.1207/s15327957pspr0803_1

Weiner, B. (1985). An attributional theory of achievement motivation and emotion.

Psychological Review, 92, 548-573. doi: 10.1037/0033-295X.92.4.548

Weiss, B., Dodge, K. A., Bates, J. E., \& Pettit, G. S. (1992). Some consequences of early harsh discipline: Child aggression and a maladaptive social information processing style. Child Development, 63, 1321-1335. doi: 10.2307/1131558

Williams, S.C., Lochman, J.E., Phillips, N.C., \& Barry, T.D. (2003). Aggressive and nonaggressive boys' physiological and cognitive process in response to peer provocations. Journal of Clinical Child and Adolescent Psychology, 32, 568-576. doi: 10.1207/S15374424JCCP3204_9

$\mathrm{Xu}, \mathrm{Y} .$, \& Zhang, Z. (2008). Distinguishing proactive and reactive aggression in Chinese children. Journal of Abnormal Child Psychology, 36, 539-552. doi: 10.1007/s10802-0079198-0

Young, L. A. (2010). Shifting our attention to encoding: Intervening at the forgotten stage of social information-processing among reactively aggressive youth. (Unpublished doctoral dissertation). The University of Alabama, Tuscaloosa, AL. 


\section{APPENDIX}

\section{Parent Debriefing Interview}

"Thank you for agreeing to participate in this study and allowing your child to be in this study. Before we begin the debriefing procedure, I would like to get your opinion about the study by asking several questions."

1. How did you feel about the component of the study in which your child was told that he would be playing someone his age, but he was really playing against the computer?

2. If another parent asked you about the study, would you encourage them to participate in it or not?

3. What part(s) did you not like?

4. What part(s) did you like?

5. During the next few minutes, I am going to explain to your child how and why he/she was told that he/she was playing another child, when it was really the computer. I will also explain that you were told about the deception at the beginning in order to make sure that it was ok with you. How do you think your child will respond? Do you have any concerns?

6. Do you have any additional questions or comments? 


\section{Home Interview with Child}

A. Pretend that you are standing on the playground playing catch with a kid named Todd/Jessica. You throw the ball to Todd/Jessica and he/she catches it. You turn around, and the next thing you realize is that Todd/Jessica has thrown the ball and hit you in the middle of your back. The ball hits you hard, and it hurts a lot.

Why do you think Todd/Jessica hit you in the back?

B. Pretend that you see some kids playing on the playground. You would really like to play with them, so you go over and ask one of them, a kid named Alan/Leah, if you can play. Alan/Leah says no.

Why do you think Alan/Leah said no?

C. Pretend that you are walking to school and you're wearing brand new sneakers. You really like your new sneakers and this is the first day you have worn them. Suddenly, you are bumped from behind by a kid named John/Lisa. You stumble into a mud puddle and your new sneakers get muddy.

Why do you think John/Lisa bumped you?

D. Pretend that you are a new kid in school and you would really like to make friends. At lunchtime, you see some kids you would like to sit with and you go over to their table. You ask if you can sit with them and a kid named Carl/Carolyn says no.

Why do you think Carl/Carolyn said no? 
E. Pretend that you go to the first meeting of a club you want to join. You would like to make friends with the other kids in the club. You walk up to some of the of the other kids and say "Hi!", but they don't say anything back.

Why do you think the other kids didn't answer you?

F. Pretend that you are walking down the hallway in school. You're carrying your books in your arm and talking to a friend. Suddenly a kid named Brett/Wendy bumps you from behind. You stumble and fall and your books go flying across the floor. The other kids in the hall start laughing.

Why do you thing Brett/Wendy bumped into you?

G. Pretend that it is your first day at school. You don't know a lot of the other kids and you would like to make friends with them. You see some kids playing a rope game so you walk up and say "Hi! 11 but no one answers you.

Why do you think the other kids didn't answer you?

H. Pretend that you and your class went on a field trip to the zoo. You stop to buy a coke. Suddenly, a kid named David/Allison bumps your arm and spills your coke all over your shirt. The coke is cold, and your shirt is all wet.

Why do you think David/Allison bumped into you?

Ambiguous Minor Harm items: A, C, F, H

Unsuccessful Peer Entry items: B, D, E, G 


\section{Parent-rating scale for Reactive and Proactive Aggression (PRPA)}

Children behave in different ways. This is the case not only in everyday situations, but they also react differently if they are confronted with annoying or stressful circumstances. Some children react impulsively and are quick to get angry, others become less emotional and react in a more calculating way.

We ask you to consider the following statements carefully. Please indicate for each statement the extent to which it applies to your child.

Response options:

Never True, Rarely True, Sometimes True, Usually True, Almost Always True

1. When my child has been teased or threatened he/she gets angry and strikes back.

2. My child gets other kids to gang up on somebody that he/she doesn't like.

3. My child is a bully.

4. My child is quick to think that others have it in for him/her.

5. My child uses physical force (or threatens to use physical force) in order to dominate other kids.

6. My child gets angry quickly if he/she does not get his or her own way.

7. My child cheats in order to win.

8. My child claims that other children are to blame in a fight and feels that they started the trouble.

9. My child is sneaky in order to gain an advantage.

10. If my child is challenged or pestered, he/she reacts immediately and impulsively.

11. My child gets angry quickly if he/she is corrected.

12. My child threatens or bullies others in order to get his/her own way.

13. My child is a bad loser.

14. My child pesters or torments younger children who are different (e.g. those who wear glasses or who have red hair).

15. My child gets angry quickly when minor things go wrong.

16. If my child is challenged or teased, he/she takes revenge in a calculating way.

17. My child uses others to get his/her way.

18. If my child does not get his/her own way, he/she reacts immediately and impulsively.

19. My child bosses others around.

20. When someone accidentally hurts my child (such as bumping into him/her), he/she assumes that the peer meant to do it and then reacts with anger/fighting.

21. If my child does not get his/her own way, he/she takes revenge in a calculating way.

22. My child is a hot head.

11-item measure - Reactive aggression items: 6, 10, 11, 15, 18, 22

Proactive aggression items: 2, 9, 12, 14, 17 
Office for Research

Institutional Review Board for the Protection of Human Subjects

ALABAMA

June 22,2011

\section{Anna Yaros}

Department of Psychology

College of Arts \& Sciences

Box 870348

Re: IRB Application \# 08-099-R3 "Measuring Children's Online Hostile Attribution Biases"

Dear Ms. Yaros:

The University of Alabama IRB has received the revisions requested by the full board on 5/20/11. The board has reviewed the revisions and your protocol is now approved for a one-year period. Please be advised that your protocol will expire one year from the date of approval, 5/20/11.

If your research will continue beyond this date, complete the renewal portions of the IRB Renewal Application. If you need to modify the study, please submit the Modification of An Approved Protocol Form. Changes in this study cannot be initiated without IRB approval, except when necessary to eliminate apparent immediate hazards to participants. When the study closes, please complete the Request for Study Closure Form.

Should you need to submit any further correspondence regarding this proposal, please include the assigned IRB application number. Please use reproductions of the IRB approved stamped consent/assent forms to obtain consent from your participants.

Good luck with your research.

Sincerely,

Stuart Usdan, $\mathrm{PhD}$.

Chair, Non- Medical Institutional Review Board

The University of Alabama 